\title{
The multi-thermal emission in solar active regions
}

\author{
G. Del Zanna
}

DAMTP, Centre for Mathematical Sciences, Wilberforce Road, Cambridge CB3 0WA, UK

e-mail: g.del-zanna@damtp.cam.ac.uk

Received 5 April 2013 / Accepted 9 July 2013

\begin{abstract}
We present simultaneous SDO AIA and Hinode EIS observations of the hot cores of active regions (ARs) and assess the dominant contributions to the AIA EUV bands. This is an extension of our previous work. We find good agreement between SDO AIA, EVE and EIS observations, using our new EIS calibration and the latest EVE v.3 data. We find that all the AIA bands are multi-thermal, with the exception of the 171 and $335 \AA$, and provide ways to roughly estimate the main contributions directly from the AIA data. We present and discuss new atomic data for the AIA bands, showing that they are now sufficiently complete to obtain temperature information in the cores of ARs, with the exception of the $211 \AA$ band. We found that the newly identified Fe XIV $93.61 \AA$ line is the dominant contribution to the $94 \AA$ band, whenever Fe XVIII is not present. Three methods to estimate the Fe XVIII emission in this band are presented, two using EIS and one directly from the AIA data. Fe XVIII emission is often present in the cores of ARs, but we found cases where it is formed at $3 \mathrm{MK}$ and not $7 \mathrm{MK}$, the temperature of peak ion abundance in equilibrium. The best EIS lines for elemental abundance determination and differential emission measure (DEM) analysis are discussed. A new set of abundances for many elements are obtained from EIS observations of hot $3 \mathrm{MK}$ loops. The abundances of the elements with low first ionisation potential (FIP), relative to those of the high-FIP elements, are found to be enhanced by about a factor of three, compared to the photospheric values. A measurement of the path length implies that the absolute abundances of the low-FIP elements are higher than the photospheric values by at least a factor of three. We present a new DEM method customised for the AIA bands, to study the thermal structure of ARs at 1" resolution. This was tested on a few ARs, including one observed during the Hi-C rocket flight. We found excellent agreement between predicted and observed AIA count rates and EIS radiances. Overall we found few differences between the AIA and Hi-C $193 \AA$ images of coronal structures, despite the higher Hi-C resolution $\left(0.25^{\prime \prime}\right)$. The Hi-C images and the AIA DEM modelling suggest that some of the cooler loops (below 1 MK) are already resolved by AIA, while the hotter (1.5-2.5 MK) "background" emission is in most places still unresolved even at the Hi-C resolution. This unresolved emission is significantly lower than previously observed with TRACE, the SOHO CDS, and Hinode EIS spectrometers. Its enhancement appears to be mostly due to increased iron abundance. We find an ubiquitous presence of emission at different temperatures that is not co-spatial, and suggest that future high-resolution imaging is carried out with isothermal bands.
\end{abstract}

Key words. atomic data - techniques: spectroscopic - Sun: corona

\section{Introduction}

Defining the thermal structure of the coronal emission in solar active regions (ARs) is a key element for our understanding of the physical mechanisms responsible for their heating. Observational and theoretical aspects have been debated for a very long time. For recent reviews, see e.g. Klimchuk (2010) and Reale (2010). This paper focuses on a few observational aspects, combining the latest imaging and spectroscopic observations with recent atomic data and modelling.

Most of the observational facts about active regions have been known since the 1970 s thanks to the Skylab observations. Key observations were made even earlier though, from the ground. Many important aspects of ARs have been found from later missions such as SMM, Yohkoh, SOHO, TRACE, and Hinode. The SOHO Coronal Diagnostic Spectrometer (CDS) has routinely provided (since 1996), spatially resolved, monochromatic images of active regions over a wide temperature range. The instrument was carefully calibrated inflight (see Del Zanna et al. 2010a). The Hinode EUV Imaging Spectrometer (EIS), with its two channels (SW: 166-212 Aं; LW: 245-291 $\AA$ ), has provided (since 2006) AR observations in a wide range of temperatures (e.g. from all the iron ionisation stages from Fe VII to Fe XVI and above 10 MK from Fe XXIII and
Fe XXIV, as presented in detail in Del Zanna 2009a; Del Zanna 2012b, 2008; Del Zanna et al. 2011b). We have also recently provided an in-flight calibration for EIS (Del Zanna 2013). These spectrometers have great temperature resolution but poor spatial (CDS: 4-5"; EIS: 3-4") and temporal resolution.

Imaging from TRACE had excellent spatial resolution (about $1^{\prime \prime}$ ) but limited temperature resolution, and significant stray light. The Atmospheric Imaging Assembly (AIA, see Lemen et al. 2012) on-board the Solar Dynamic Observatory (SDO), launched in 2010, has been routinely observing the solar corona at high resolution (about $\left.1^{\prime \prime}\right)$ and high cadence (12 s) with six EUV bands. In principle these observations allow the study of the thermal structure of active regions at high spatial resolution. Another significant advance compared to previous instruments is the low stray light, which has been recently assessed by Poduval et al. (2013).

The highest-resolution images (about $0.25^{\prime \prime}$ ) of the solar corona have recently been taken with an excellent rocket borne telescope with a broad band similar to AIA $193 \AA$ (Cirtain et al. 2013). These images provide important information on the small-scale structures in ARs.

Active regions are notoriously bright in the X-rays in their cores. The Skylab X-ray imaging suggested that the long-lasting "hot" core loops in quiescent ARs are approximately isothermal 
at around 3 MK (Rosner et al. 1978), which was a puzzling feature. Later spectroscopic measurements in the X-rays from SMM/FCS confirmed that most active region cores were nearly isothermal, at least in the sense that very little material at temperatures above $3 \mathrm{MK}$ is present (see, e.g. Schmelz et al. 1996). EUV spectroscopy with SOHO CDS also confirmed this (Del Zanna \& Mason 2003). For simplicity, we call these hot core loops " $3 \mathrm{MK}$ " loops, bearing in mind that the isothermality of these $3 \mathrm{MK}$ loops is not universally accepted. Various recent studies based on Hinode EIS observations discussed AR cores (e.g. Tripathi et al. 2011; Warren et al. 2012) but did not analyse in detail the thermal structure of the 3 MK loops. Winebarger et al. (2011) calculated the differential emission measure (DEM) of the apex region of the hot core loops, and found the DEM to be broad and peaked around $3 \mathrm{MK}$.

We note that previous studies used the Hinode EIS ground calibration, hence need to be revised in light of our new calibration (Del Zanna 2013), which mainly increases (by about a factor of two) the intensities of the lines in the LW channel. This affects the emission measures at $3 \mathrm{MK}$, since they are constrained by the strong Fe XIV-Fe XVI lines observed in this channel. We also note that, in general, it is not straightforward to determine how isothermal a plasma is, with significant uncertainties originating from the accuracy of the observations and atomic data (see, e.g. Judge 2010; Landi \& Klimchuk 2010).

One of the aims of this paper is to study the thermal structure of these $3 \mathrm{MK}$ loops taking into account the new EIS calibration, a different selection of lines, a discussion of elemental abundances, and background/foreground subtraction, all aspects that have often been neglected in the literature, but that turn out to be quite important. These loops appear "fuzzy", i.e. unresolved at $1^{\prime \prime}$ resolution. This has been known since Skylab X-ray imaging, EUV imaging from TRACE (see, e.g. Brickhouse \& Schmelz 2006), and actually for a long time from ground-based observations of the coronal forbidden lines at visible wavelengths (e.g. in $\mathrm{Ca}$ XV). However, detailed measurements of densities and abundance have been lacking from the literature.

In the EUV, the brightest emission in ARs comes from lowlying emission (commonly known as "moss") that was clearly visible with high-resolution imagers observing $1 \mathrm{MK}$ emission such as NIXT (Peres et al. 1994) and TRACE $173 \AA$ passband (see, e.g. Schrijver et al. 1999). Martens et al. (2000) proposed that moss is the transition-region emission of the 3 MK loops, a view now commonly accepted. We note however that moss emission is co-spatial with all plage regions, and plage regions are the magnetic footpoint of all coronal structures, i.e. not only the 3 MK loops. Various studies on moss emission have been performed, to try and gain an insight into the physics of the 3 MK loops (see, e.g. Tripathi et al. 2010, 2012; Winebarger et al. 2011). We will discuss moss characteristics in a separate paper, but note that any measurement of coronal structures needs to avoid regions where moss is present.

Active regions, especially during the later stages of their lives, produce long-lasting "warm" loops, formed between 1 and 2 MK, spectacularly observed with the TRACE $173 \AA$ (Fe IX) band. The thermal characteristics of these loops have been debated for a very long time. Clearly, EUV imaging from TRACE had limited temperature information. Until 2003, it was commonly thought that the majority of AR loop structures were multi-thermal, and that most cooler loops were actually hot loops observed during the cooling process.

Our SOHO CDS observations, combined with TRACE high-resolution imaging of many ARs actually showed a very different picture. Most warm loops were found to be nearly isothermal in their cross-section, with filling factors close to one at their base (Del Zanna 2003a; Del Zanna \& Mason 2003; Cirtain et al. 2007). Our results have so far been largely confirmed by Hinode EIS and SDO AIA observations. For a few examples, see e.g. Warren et al. (2008), Tripathi et al. (2009), Aschwanden \& Boerner (2011), Schmelz et al. (2013), although some loops show some departures (Schmelz et al. 2009, 2010), and the issue is still debated.

In summary, our view is that a significant fraction of the coronal emission in active regions is composed of loop structures that are nearly isothermal in their cross-section, hence are better observed with spectrometers with high spatial resolution and/or with monochromatic imagers. Clearly, given the limited spatial resolution of current spectrometers, most loop structures appear as multi-thermal, due to line of sight effects. It is thought that individual magnetic flux tubes are isothermal, and that resolved loops are composed of a superposition of such elementary structures (Klimchuk 2006). Measuring how isothermal AR loops are, is therefore a useful observational constraint for loop modelling.

The effective spatial resolution of EIS is only marginally better than CDS. The AIA resolution is excellent, but one obvious question we want to address here is whether the nature, the calibration and the atomic data for the AIA EUV bands are accurate enough to measure the thermal structure of active regions.

As we showed in O'Dwyer et al. (2010) and Del Zanna et al. (2011c), the AIA EUV bands are significantly multi-thermal, which naturally "blurs" their view of active region loops and limits their use. The O'Dwyer et al. (2010) study was purely theoretical, while the Del Zanna et al. (2011c) study combined Hinode EIS and AIA observations to show that significant "cool" $(T<1 \mathrm{MK})$ emission is present in all the AIA EUV bands. EIS has a significant temperature overlap with the AIA bands, and also directly observes some of the AIA bands, so it is an excellent instrument for this purpose. We highlighted several problems in the AIA and EIS data, but also in the atomic data, especially for the $211 \AA$ band, where up to a half of the emission can come from unidentified spectral lines. In this paper we aim to extend the previous work to discuss the various contributions to the EUV AIA bands in the case of hot emission (cores of active regions), and to see how the AIA data can be used to study the AR multi-thermal structure with DEM modelling.

Our SOHO CDS observations also suggested the presence of a pervasive and dominant unresolved emission in the 1.5-2.5 MK temperature range (Del Zanna \& Mason 2003), which constituted about $80 \%$ of the EUV AR emission observed by CDS and TRACE, and was neglected in previous analyses. Similar levels of emission are also observed with EIS, but not with AIA as we shall discuss here. Another natural question we want to address here is whether this unresolved emission is real or is an artifact of the low spatial resolution of the spectrometers and stray light in the imagers.

\section{AIA and EIS observations}

We have analysed several SDO/AIA and Hinode/EIS sets of observations, for both on-disk and off-limb active regions. We used an EIS study (Atlas_60) that we designed for the purpose of the cross-calibration between AIA and EIS. Here, we present a sample of results from two on-disk active regions, observed on 2010 Nov. 23 and 2010 Oct. 26. The first one was discussed in Del Zanna et al. (2011c) as a clear example of a case when significant cool emission is present in all the AIA EUV bands. 
It is also a clear case where hotter emission from Fe XVIII is not present in the $94 \AA$ band. The second one is in contrast a clear case where Fe XVIII is present in the $94 \AA$ band. We also analysed an AR observation of 2010 Dec. 11 at the limb, where some Fe XVIII emission is present in the core.

The EIS observations consist of single rastering of the $2^{\prime \prime}$ slit (from west to east) 60 times by $\simeq 2^{\prime \prime}$ steps, for a field-ofview (FOV) of $120^{\prime \prime} \times 140^{\prime \prime}$. With $60 \mathrm{~s}$ exposure time, the observation lasted $\simeq 60 \mathrm{~min}$. Details on the complex processing and correlation of the EIS and AIA data can be found in Del Zanna et al. (2011c). Various geometrical corrections were applied to both AIA and EIS. The AIA plate scale was adjusted for each observation by examining the solar limb, and then cross-checked by cross-correlating low-lying features such as the moss. The co-alignment is good to one AIA pixel. The AIA images were then reduced to the lower spatial and temporal resolution of the EIS data for a direct comparison. The AIA images were convolved with a Gaussian of $2^{\prime \prime}$ full-widthhalf-maximum (FWHM). Various comparisons on different ARs were performed, and the results consistently indicate an effective resolution of EIS of about 3-4". This is due to a superposition of various effects which include the intrinsic EIS point spread function, its stray light, and the instrument and satellite jitter during each exposure. The long exposures required for a spectrometer, combined with the intrinsic AR variability, also contribute to a "blurring" effect.

\section{Atomic data and instrument calibrations}

In the past few years, we (the APAP team ${ }^{1}$ ) have produced a significant amount of new large-scale atomic calculations for a large number of ions, including Fe VII (Witthoeft \& Badnell 2008), Fe VIII and Fe IX (O'Dwyer et al. 2012), Fe X (Del Zanna et al. 2012b), Fe XI (Del Zanna \& Storey 2013), Fe XII (Del Zanna et al. 2012a), Fe XIII (Del Zanna \& Storey 2013), Fe XIV (Liang et al. 2010), and Fe XVI (Liang et al. 2009). The R-matrix calculations for Fe X-Fe XIII were not trivial, and a lot of surprising issues were found. The main issue was that cascading and resonance excitations are extremely important for some of the soft X-ray and EUV lines. These atomic data have been benchmarked against the best solar and laboratory observations in a series of papers, where several new identifications have been provided. The review of the soft X-rays is given in Del Zanna (2012a). For the present analysis we adopt the above atomic data and line identifications, and CHIANTI v.7 for the other ions. Note that some of the new data and identifications have already been made available to CHIANTI v.7.1 (the Fe VIII, Fe IX and Fe XIV atomic data, and some of the Del Zanna 2012a identifications). The latest v.7.1 CHIANTI ionisation equilibrium tables (Landi et al. 2013) are adopted here. A notable feature is a change in the ionisation equilibrium for Fe VIII and Fe IX, compared to the previous one (Dere et al. 2009).

During the present analysis, significant discrepancies in the radiances of the EIS lines were found, with most lines in the long-wavelength (LW) channel being a factor of two too weak, compared to those in the short-wavelength (SW) band, if the ground calibration is adopted. A detailed analysis has led to a new time- and wavelength-dependent radiometric calibration (Del Zanna 2013) which is adopted here. The new calibration increases all the LW lines by about a factor of 2 for the observation discussed here, but leaves those of the SW band substantially

\footnotetext{
http://www . apap-network. org
}

unchanged (in agreement with the in-flight calibration discussed in Wang et al. 2011).

An assessment of the AIA calibration for data in 2010 Dec. 10 has been done by comparing the SDO Extreme ultraviolet Variability Experiment (EVE; Woods et al. 2012) and AIA observations. We compared the full-disk AIA data numbers (DN) during the first hour of 2010 Dec. 10 with the values estimated directly from EVE, using the AIA pre-flight calibration of Boerner et al. (2011). We measured the radial distribution of the off-limb AIA count rates and estimated the missing AIA off-limb contribution to be negligible (of the order of 1\%). With the EVE v. 2 data, we obtained the following correction factors to the AIA effective areas: $0.79,0.84,1.28,1.13,1.12$ for the $131,171,193$, 211, and $335 \AA$ bands. The correction factors suggested within the AIA software (keywords evenorm and timedepend_date) are very similar $(0.78,0.83,1.24,1.09,1.08)$. However, with the recent EVE v.3 calibration, we obtained different values: 0.83, $1.11,1.12,0.97,1.0$. These corrections have not been applied, since they are well within the uncertainties of the EVE calibration $(25 \%)$. We also note that a proper in-flight AIA calibration is non-trivial. For example, any correction to the 94 and $171 \AA$ bands has an additional uncertainty due to the low EVE spectral resolution.

We used the present atomic data and the pre-flight effective areas (Boerner et al. 2011) to calculate the AIA response functions, following the simple methods described in Del Zanna et al. (2011c). These responses are basically the sum of the emissivities of the lines and continua folded with the effective areas and summed over wavelength. We calculated the responses at a constant electron density of $2 \times 10^{9} \mathrm{~cm}^{-3}$, typical for the upper transition-region emission in warm loops (Del Zanna \& Mason 2003), but note that some variations would be present if different densities are used. Note that the AIA response functions available within Solarsoft assume a constant pressure of $10^{15} \mathrm{~cm}^{-3} \mathrm{~K}$ and a set of "coronal" elemental abundances.

We adopted a set of "coronal" abundances discussed below, where most low first ionisation potential (FIP) elements such as iron have been increased by a factor of 3.16. The curves are shown in Fig. 1. We also calculated the responses with the photospheric abundances of Asplund et al. (2009). These values, increased by 3.16 , are also shown in the figure. The peaks agree very well because the dominating lines in all the AIA EUV bands are from iron, so the abundances are just a scaling factor, although some differences are present in the continuum. This means that emission measure modelling with AIA is largely independent of elemental abundances. Finally, the dot-dash lines in Fig. 1 show the responses calculated with CHIANTI v.7.1.

\subsection{AIA $131 \AA$}

The AIA $131 \AA$ band is dominated by the two Fe VIII lines at 130.94 and $131.24 \AA$ although significant contribution in ARs comes from a large number of lines, plus the free-bound continuum. The atomic data for Fe VIII are uncertain. As described in Del Zanna (2009b), adjustments to the Griffin et al. (2000) atomic data were necessary to improve the EUV lines observed by Hinode EIS. These adjustments, implemented in CHIANTI v.7, were based on a large atomic structure calculation that focused on the lower levels and was not accurate for the $4 \mathrm{f}$ levels that are producing the lines in the AIA $131 \AA$ band. A recent large-scale atomic calculation from Tayal \& Zatsarinny (2011) did not resolve the problems with the EUV lines (cf. their Table 4), but should in principle provide better atomic data for 

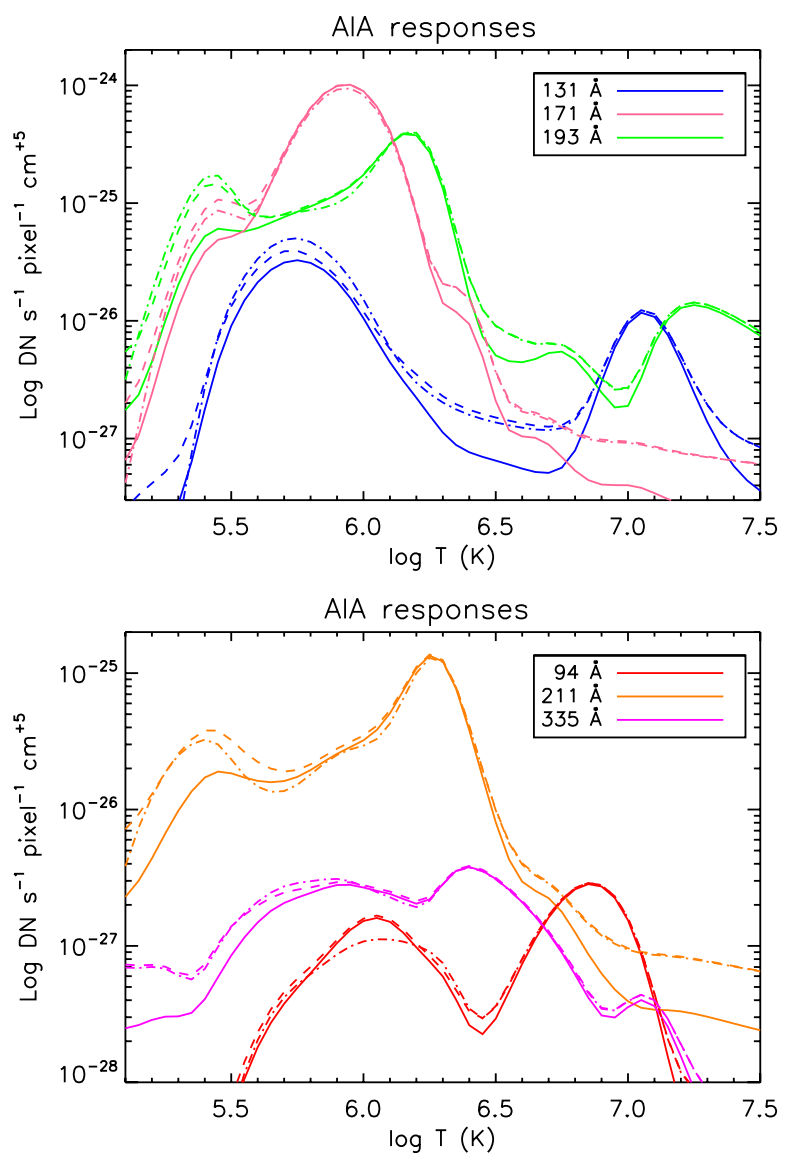

Fig. 1. SDO AIA response functions calculated with the present atomic data, the present "coronal" abundances and a constant electron density of $2 \times 10^{9} \mathrm{~cm}^{-3}$ (full lines). The dashed lines are the responses calculated with "photospheric" abundances, multiplied by 3.16. The dash dot lines are calculated with CHIANTI v.7.1, and "photospheric" abundances multiplied by 3.16 .

the $4 \mathrm{f}$ lines. We therefore built a new ion model where we used the Tayal \& Zatsarinny (2011) data but also applied some corrections, based on the Del Zanna (2009b) calculations for the levels numbered 41, 43, 45, and 46. Tayal \& Zatsarinny (2011) only provided some A values for the dipole-allowed transitions, however a significant number of $\mathrm{A}$ values for forbidden transitions are necessary to redistribute the level population, especially to de-populate the metastable levels for this ion. The values calculated by Del Zanna (2009b) have been added to those provided by Tayal \& Zatsarinny (2011). The new ion model produces a significant (about 40\%) decrease in the $131 \AA$ band response, as shown in Fig. 1.

When flare-like emission is present, significant continuum and Fe XXI (formed around $10 \mathrm{MK}$ ) emission is present in the $131 \AA$ band, with some contribution also from Fe XX and Fe XXIII (see Petkaki et al. 2012; Del Zanna \& Woods 2013). This hot contribution significantly complicates the use of this band for DEM analyses, because it is typically superimposed on the cooler (mostly Fe VIII) emission, for which we have no other means of establishing the amount. Looking at many ARs, we found that the Fe XXI is only occasionally produced in the same region as Fe XVIII, observed in the $94 \AA$ band (see below). This means that the estimated Fe XVIII cannot be used as a proxy to estimate the Fe XXI emission. Also, the DEM that can be estimated from Fe XVIII is not necessarily the same as that for Fe XXI. So, as a first approximation, we did not use the $131 \AA$ band for the DEM analysis for all the regions where the estimated Fe XVIII emission is above a threshold value. In the case of obvious flaring and Fe XXI contribution, the $131 \AA$ band is used for the DEM analysis.

\section{2. $A I A 171 \AA$}

The $171 \AA$ band turns out to be always dominated by the resonance transition from Fe IX, for which the atomic data of Storey et al. (2002) are expected to be reliable. This line is predicted to be sensitive to both the temperature and density structure of the emitting plasma. As shown in Del Zanna et al. (2011c), this line is formed over a very broad temperature range $(\log T[\mathrm{~K}]=5.5-$ 6.2). In the cores of active regions, some blending (about $10 \%$ of the Fe IX) is due to Ca XV 171.6 $\AA$.

The $171 \AA$ line is barely visible in the EIS spectra, being in a region where the EIS sensitivity is about three orders of magnitude lower than at the peak around $195 \AA$. A direct AIA vs. EIS comparison is therefore difficult.

\subsection{AIA and Hi-C $193 \AA$}

This band is relatively well understood, because all the lines are observed with EIS, and since 2004 we have made a significant effort to identify the lines and provide accurate atomic data especially for Fe X, Fe XI, and Fe XII. We note that the strongest lines in this band are from Fe XII. When flare-like emission is present in the cores of ARs, the CaXVII $192.8 \AA$ and Fe XXIV $192.0 \AA$ lines provide a significant contribution to this band. Near the footpoints of warm loops, Fe VII, Fe VIII, and Fe IX produce a significant contribution (Del Zanna et al. 2011c). We note that Fe VII is not included in CHIANTI yet because of uncertainties in the identifications of the strongest lines. Here, we adopt the identifications of Del Zanna (2009a). Some unidentified lines are also present in this band (Del Zanna et al. 2011c), although they contribute little in most AR areas.

Despite common belief, only about half of the counts recorded in the AIA $193 \AA$ band in the cores of active regions are due to the Fe XII lines. The rest of the counts are due to several transitions formed over a broad range of temperatures. There is significant contribution from Fe XI, also from Fe X and Fe XIII. We have discovered this by comparing the simultaneous AIA and EIS observations of a few ARs. Figure 2 shows as an example the AR observed on-disk on 2010 Nov 23. Its top right image shows the total AIA count rates due to the Fe XII 186.8, 192.4, 193.5, and 195.1 A lines, all observed by EIS. These count rates were obtained by converting the EIS calibrated radiances into AIA DN/s. They should be compared to the total AIA count rates, shown in the top left image. By comparing the AIA count rates due to Fe XII (as estimated by EIS) with the AIA images, we found that the DN/s due to all the Fe XII lines in the $193 \AA$ band can be roughly estimated in this way:

$I(\mathrm{Fe}$ XII $)=0.7 \times(I(193 \AA)-I(171 \AA) / 2)$

Fig. 2 shows (middle top) an image of the AIA DN/s due to the Fe XII lines as obtained from this expression. A comparison with the measured Fe XII intensity shows that this linear relation overestimates the Fe XII contribution in the areas with strong cool emission and little Fe XII emission, as for example pixel position 25 in the lower panel of Fig. 2.

We note that for the present DEM analysis the total observed and predicted emission in this band has been used, since the cooler contribution to this band can be estimated quite accurately 
G. Del Zanna: The multi-thermal emission in solar active regions
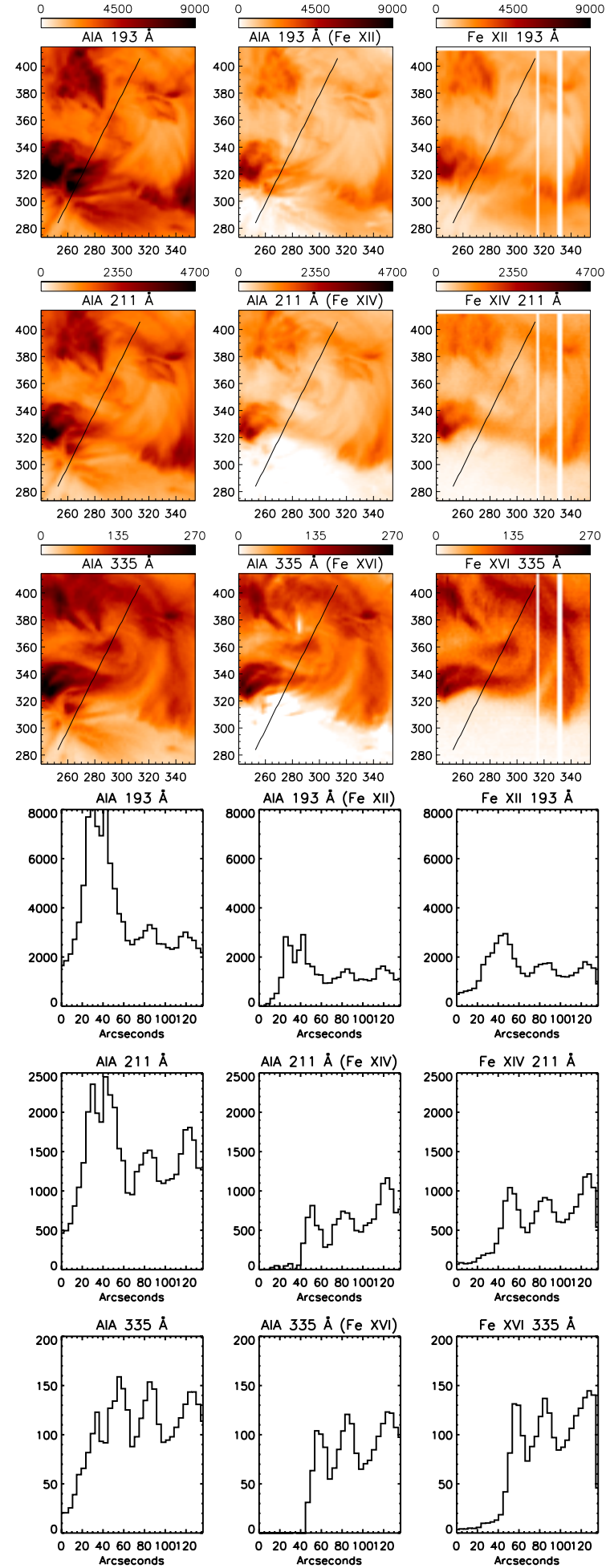

Fig. 2. Top three left images: AIA count rates (DN/s) of the on-disk AR observation of 2010 Nov. 23, rebinned to the EIS resolution. Top three middle images: estimated AIA count rates due to Fe XII, Fe XIV, and Fe XVI (see text). Top three right images: measured Fe XII, Fe XIV and estimated Fe XVI (in AIA DN/s) directly from EIS. The axes in the images indicate arc seconds from Sun centre. The bottom panels show the corresponding profiles (AIA DN/s) along the segment indicated in the images. The AR core spans the 60-130 arcsec region in the profiles.

with the 131 and $171 \AA$ A bands, and the atomic data for the $193 \AA$ band are relatively complete.

\subsection{AIA $211 \AA$}

The $211 \AA$ AIA band is the least understood, with a significant number of lines not yet identified, as discussed in Del Zanna et al. (2011c). Some of them are "cool" lines (Del Zanna 2009a), while others are coronal (Del Zanna 2012b). The main coronal line is the Fe XIV 211.3 $\AA$ transition. This Fe XIV line is observed directly by Hinode/EIS, so we can estimate precisely its contribution to the AIA band (assuming that the EIS and AIA calibrations are correct). Figure 2 (right middle image) shows the AIA DN/s due to the Fe XIV $211.3 \AA$ line. In the cores of ARs, only about $60 \%$ of the counts in the $211 \AA$ band come from the Fe XIV transition at $211.3 \AA$.

The missing atomic data for this band means that it cannot be used as it is for DEM analyses. In the absence of EIS observations, the AIA $211 \AA$ DN/s due to the Fe XIV line can be roughly estimated by subtracting a linear combination of the 171 and $193 \AA ̊$ bands

$$
I(\mathrm{Fe} \mathrm{XIV})=I(211 \AA)-I(171 \AA) / 17-I(193 \AA) / 5 .
$$

The result obtained from this relation is shown in Fig. 2 (middle image). We have used this linear combination as input for all the DEM analyses presented in this paper. The corresponding response for this band was calculated by adding the Fe XIV emissivity to the continuum. The atomic data for Fe XIV are reliable (Liang et al. 2010; Del Zanna 2012b) and the $211.3 \AA$ line does not have significant temperature or density effects.

\subsection{AIA $335 \AA$}

The $335 \AA$ band is also highly multi-thermal, although the dominant contribution in the cores of active regions is from a single Fe XVI $335.4 \AA$ transition, for which the atomic data (Liang et al. 2009) are reliable. We have checked this by estimating the Fe XVI $335.4 \AA$ A line using the intensity of the Fe XVI $263 \AA$ line observed by EIS, and adopting a theoretical ratio of 17.5 (photons) for the two lines. Figure 2 (bottom right image) shows the estimated AIA $335 \AA$ DN/s due to the Fe XVI $335.4 \AA$ line. The residual emission in AR cores comes from a host of transitions and ions (Fe XIV, Mg VIII, Fe XII, etc.). We found that the empirical relation

$$
I(\mathrm{Fe} \mathrm{XVI})=I(335 \AA)-I(171 \AA) / 70,
$$

provides a rough estimate for the AIA DN/s due to the Fe XVI $335.4 \AA$ line in the AR cores. Figure 2 (middle lower image) shows the result of this linear relation. This empirical adjustment sometimes leaves a small residual due to the cooler lines in regions where the Fe XVI $335.4 \AA$ line is very weak.

We have used this estimate as input in some DEM analyses, using for the response function just the Fe XVI $335.4 \AA$ contribution function, added to the continuum. This procedure effectively removes the double peak for this band. The lower temperature peak in this band is due to significant TR emission, as discussed in Del Zanna et al. (2011c) (see the cool loops in the bottom left of the images in Fig. 2).

\subsection{AIA $94 \AA$}

This band is particularly important for the presence of the Fe XVIII $93.932 \AA$ line. Significant problems in the $94 \AA$ band were reported by several authors (see, e.g. Aschwanden \& Boerner 2011; Del Zanna et al. 2011c; Schmelz et al. 2011; Foster \& Testa 2011; Testa et al. 2012b), but it was not clear 
if these were due to calibration or atomic data issues, so a few authors produced empirical estimates for the Fe XVIII emission (Reale et al. 2011; Testa \& Reale 2012; Warren et al. 2012).

When the Fe XVIII emission becomes dominant, we found good agreement between observations and theory (Petkaki et al. 2012), suggesting that the AIA $94 \AA$ calibration is correct, given that the atomic data we provided for Fe XVIII should be reliable (Witthoeft et al. 2006; Del Zanna 2006).

Outside of obvious flaring emission, the $94 \AA$ band was thought to be dominated by Fe X $94.012 \AA$, identified by Edlén (1937). Del Zanna et al. (2011c) however pointed out that at least two other unidentified components were present in ARs, one "cooler" below $1 \mathrm{MK}$, and one "hotter" around 2-3 MK.

The main problem for this band was the lack of atomic data and line identifications in the soft X-rays. As already mentioned, we have performed new large-scale $\mathbf{R}$-matrix calculations for several iron ions, to provide the data for the soft X-ray lines. The soft X-rays (which include this band) were particularly problematic because the majority of lines were unidentified. These new atomic data have now been benchmarked against soft X-ray solar and laboratory observations in Del Zanna (2012a), where several new identifications (for all the strongest lines) were provided. Of all these new identifications, the Fe XIV 93.61 $\AA$ transition is of particular importance for AR observations in the AIA $94 \AA$ band, because it becomes very strong. Indeed, it has been known for a very long time (see, e.g. Widing \& Sandlin 1968; Manson 1972) that when the Sun is active (because of the presence of non-flaring ARs) the irradiance of the $93.61 \AA$ line becomes almost as strong (66\%) as that of the Fe X 94.012 $\AA$ transition. Clearly, this line must become stronger than Fe $\mathrm{X}$ in AR radiance observations. Note that the atomic data for Fe XIV (Liang et al. 2010) are reliable, and that the new identifications has been made available to version 7.1 of the CHIANTI database (Landi et al. 2013).

The Fe $\mathrm{X} 3 \mathrm{~s}^{2} 3 \mathrm{p}^{5}{ }^{2} \mathrm{P}_{3 / 2}-3 \mathrm{~s}^{2} 3 \mathrm{p}^{4} 4 \mathrm{~s}^{2} \mathrm{D}_{5 / 2} 94.012 \AA$ transition, identified by Edlén (1937), is the dominant emission in quiet Sun conditions. The atomic calculations for the Fe X $3 s^{2} 3 p^{4} 4 s$ levels were extremely complex, as discussed in Del Zanna et al. (2012b). Our R-matrix large-scale calculations should be accurate to within $30 \%$. Distorted wave (DW) calculations significantly underestimate the Fe $\mathrm{X}$ lines. CHIANTI version 7.1 (Landi et al. 2013) included DW data (Landi \& Dere 2013). These latter calculations predict the presence of a $3 \mathrm{~s}^{2} 3 \mathrm{p}^{5}{ }^{2} \mathrm{P}_{3 / 2}-$ $3 \mathrm{p}^{6} 3 \mathrm{~d}^{2} \mathrm{D}_{5 / 2}$ transition, at an estimated wavelength of $94.27 \AA$. There are two problems with this line. First, its intensity is predicted to be more than half the $94.012 \AA$ line, while there are no lines in astrophysical or laboratory spectra around that wavelength. Second, the position of the $3 \mathrm{p}^{6} 3 \mathrm{~d}^{2} \mathrm{D}_{5 / 2}$ level affects the $3 \mathrm{~s}^{2} 3 \mathrm{p}^{4} 4 \mathrm{~s}^{2} \mathrm{D}_{5 / 2}$ one, drastically reducing the weighted oscillator strength of the $94.012 \AA$ transition to a value of 0.13 , compared to a value of 0.31 obtained from the large-scale RM4 calculation presented in Del Zanna et al. (2012b). The benchmark of the Fe X soft X-ray lines presented in Del Zanna (2012a) shows very good agreement between the RM4 model and observations, suggesting problems in the target used in Landi \& Dere (2013).

A significant contribution from the continuum (mostly due to free-bound emission at $3 \mathrm{MK}$ ) is present in the AIA $94 \AA$ band (as discussed below), although the amount of the contribution depends on the absolute abundances.

Weak Fe VIII and Fe IX transitions are also present in this band. The Fe VIII lines were observed in the laboratory and DW data were calculated (O'Dwyer et al. 2012) and included in CHIANTI version 7.1. DW calculations for the Fe IX lines

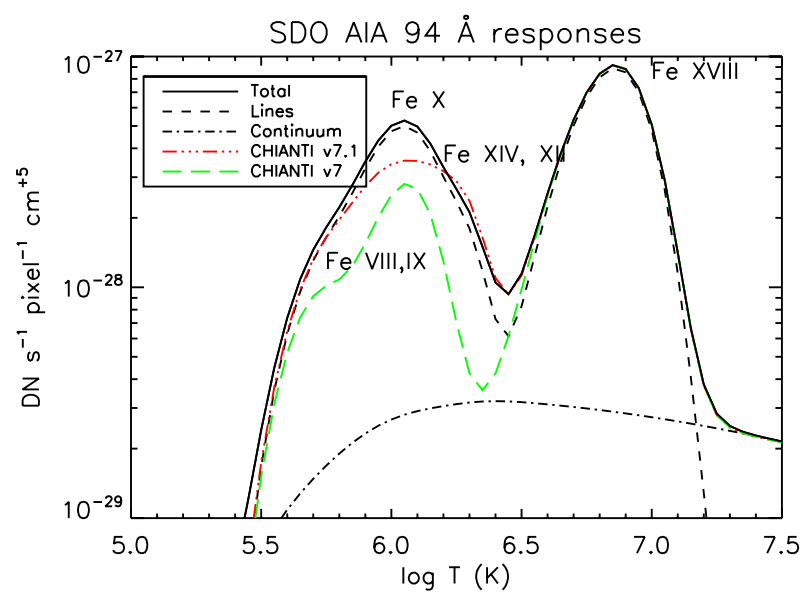

Fig. 3. AIA $94 \AA ̊$ responses.

(O'Dwyer et al. 2012) were also included in CHIANTI version 7.1, however the wavelengths of these lines are very uncertain. The benchmark work of Del Zanna (2012a) found problems in the suggestions given in O'Dwyer et al. (2012) (included in CHIANTI version 7.1), providing new recommended values.

CHIANTI version 7.1 also predicts a few Fe XII lines that could contribute to the $94 \AA$ AIA band, in particular a $3 s 3 p^{4}{ }^{2} D_{5 / 2}-3 s^{2} 3 p^{2} 4 p^{2} D_{5 / 2}$ transition. In this case, both the DW calculations of Landi \& Dere (2013) and the large-scale R-matrix one (Del Zanna et al. 2012a) agree. The estimated wavelength in CHIANTI version 7.1 is $93.68 \AA$, while that one estimated by Del Zanna (2012a) is $93.49 \AA$. Note, however, that this wavelength has a significant uncertainty, considering that large discrepancies in the energies of the $3 \mathrm{~s}^{2} 3 \mathrm{p}^{2} 4 \mathrm{p}$ levels have been found (Del Zanna 2012a).

Figure 3 shows the AIA $94 \AA$ responses calculated with the present data, together with previous CHIANTI versions. The increase around $\log T[\mathrm{~K}]=6.3$ is mainly due to the Fe XIV 93.61 line. The main difference with CHIANTI v.7.1 is due to different atomic data for Fe X. We note that various comparisons with observations suggest that the new atomic data (which will be available in version 8 of CHIANTI) are relatively accurate and complete. The use of semi-empirical fudge factors such as the /chiantifix keyword within the AIA software is discouraged.

In conclusion, the AIA $94 \AA$ band is so multi-thermal that it is not very useful for DEM analyses as it is. However, we have used it to constrain the DEM at higher temperatures, when significant Fe XVIII is present. We searched for a linear combination of the 171 and $211 \AA$ bands that would produce similar count rates as those we estimated to be due to Fe XVIII (as described below). We found that a rough estimate of the AIA $94 \AA \mathrm{DN} / \mathrm{s}$ due to Fe XVIII is

$$
I(\mathrm{Fe} \text { XVIII })=I(94 \AA)-I(211 \AA) / 120 .-I(171 \AA) / 450 .
$$

The $211 \AA$ A emission is used as a proxy for the Fe XIV (recall that the Fe XIV $211.3 \AA$ transition produces about half of the count rates) and the cooler ( $1 \mathrm{MK})$ emission. The $171 \AA$ emission is instead used to roughly estimate the weaker contributions from Fe VIII, Fe IX, and Fe X lines.

The estimated AIA Fe XVIII count rates are similar to those obtained using the Warren et al. (2012) 171, $193 \AA$ combination (note that the sum in the paper should be from 0 to 3 ), as shown below. Note that the Warren et al. (2012) empirical combination produced a good agreement with the observed Fe XVIII emission 
(Teriaca et al. 2012) for one sample AR. The agreement between the present and the Warren et al. (2012) method is not surprising, considering that the morphology of the 193 and $211 \AA$ bands is very similar in all active region cores.

\section{DEM inversion methods}

We recall that the intensity of an optically thin line can be expressed as an integral along the line of sight

$I\left(\lambda_{j i}\right)=\int_{h} N_{\mathrm{e}} N_{\mathrm{H}} A b G\left(N_{\mathrm{e}}, T, \lambda_{j, i}\right) \mathrm{d} h$

where $\mathrm{Ab}$ is the elemental abundance, $G\left(N_{\mathrm{e}}, T, \lambda_{j, i}\right)$ is the contribution function of the spectral line. $G(N, T)$ has a very strong dependence on temperature, through the ionisation balance calculations, and normally a negligible dependence on the electron number density, $N_{\mathrm{e}}$, for a certain range of densities, if the appropriate line is chosen. In the case of a broad-band instrument such as AIA, the contribution function is replaced by the instrument response, which is calculated by summing all the $G(N, T)$ of the lines in the passband and adding the emissivity of the continuum.

If a unique relationship exists between $N_{\mathrm{e}}$ and $T$, neglecting density effects we define

$\operatorname{DEM}(T)=N_{\mathrm{e}} N_{\mathrm{H}} \frac{\mathrm{d} h}{\mathrm{~d} T} \quad\left[\mathrm{~cm}^{-5} \mathrm{~K}^{-1}\right]$

as the column DEM of the plasma, which gives an indication of the amount of plasma along the line of sight that is emitting the radiation observed at a temperature between $T$ and $T+\mathrm{d} T$. The DEM by definition is a continuous distribution in a range of temperatures. We normally write

$I\left(\lambda_{i j}\right)=A b \int_{T} G(T) \operatorname{DEM}(T) \mathrm{d} T$,

assuming that the abundance of the element $A b$ is constant along the line of sight. There is an extended literature on the subject of inversion techniques and the non-uniqueness of the solution (see e.g. Craig \& Brown 1976, 1986; Judge et al. 1997 and references therein). A few specific issues are discussed in Del Zanna (1999); Del Zanna et al. (2002).

The column emission measure $E M_{h}(T, \Delta T)$ can be calculated by integrating the DEM over the temperature bins $\Delta T=$ $T 2-T 1$

$$
E M_{h}(T, \Delta T) \equiv \int_{h 1}^{h 2} N_{\mathrm{e}} N_{\mathrm{H}} \mathrm{d} h=\int_{T 1}^{T 2} \operatorname{DEM}(T) \mathrm{d} T \quad\left[\mathrm{~cm}^{-5}\right]
$$

The total emission measure $E M_{h}(T)$ is obtained by integrating over the entire temperature range.

Note that the absolute values of the DEM or $E M_{h}$ depend on the radiometric calibration of the instrument providing the intensities and the "absolute" (i.e. relative to hydrogen) values of the elemental abundances. Also note that there is a vast literature on the subject of photospheric and coronal abundances. Only a few key issues are mentioned here. The standard photospheric abundances of many elements have recently changed significantly. The low-FIP $(\leq 10 \mathrm{eV})$ elements are more abundant than highFIP in the slow solar wind (SSW) and in coronal loop plasma. Their relative values compared to hydrogen is still debated, as well as the amplitude of the difference. For recent reviews see, e.g. Asplund et al. (2009); Lodders et al. (2009); Schmelz et al. (2012). A common set of "coronal" abundances used by many authors is that one of Feldman et al. (1992), where the low-FIP elements are increased by a factor of about 4 . This set of abundances is in need of a significant revision, mainly because many values were measured relative to the photospheric abundances of high-FIP elements (such as oxygen), which have changed. Also, we note that it is now quite clear that a single set of elemental abundances cannot be representative of all the features of the solar corona. For example, the quiet Sun corona shows pretty much photospheric abundances. A strong FIP effect was thought to be present in warm loops, however a revision of the older measurements, together with SOHO CDS measurements has showed that in many cases warm loops have abundances close to the photospheric ones, i.e. the FIP enhancement was often overestimated in the literature (see Del Zanna 2003b). We discuss the abundances in hot core loops below.

An interesting measurement is obtained once the column emission measure and the average electron density $\left\langle N_{\mathrm{e}}\right\rangle$ are known. This is the path length

$\Delta h=\frac{E M_{h}}{0.83\left\langle N_{\mathrm{e}}\right\rangle^{2}} \quad[\mathrm{~cm}]$

i.e. the length along the line of sight of the emitting plasma, assuming that it is uniformly distributed. 0.83 is the value of $N_{\mathrm{H}} / N_{\mathrm{e}}$ (which depends slightly on the elemental abundances). The average electron density $\left\langle N_{\mathrm{e}}\right\rangle$, obtained from density-sensitive line ratios, is independent of the elemental abundances, and largely independent of the ionisation state of the plasma. Another parameter often used is the spectroscopic filling factor, which is obtained from the path length once the size (along the line of sight) of the plasma is estimated. This size can be estimated quite accurately only in some cases, such as clearly defined coronal loops.

One useful parameter related to the DEM is the effective temperature

$T_{\text {eff }}=\int G(T) \operatorname{DEM}(T) T \mathrm{~d} T / \int G(T) \operatorname{DEM}(T) \mathrm{d} T$

that is an average temperature more indicative of where a line is formed. This is often quite different than $T_{\max }$, the temperature where $G(T)$ has a maximum.

\subsection{DEM spline method using Hinode EIS lines}

For a few sample cases based on EIS lines we have used the method described in Del Zanna (1999), where the DEM is assumed to be a spline function. The DEM is well constrained up to $\log T[\mathrm{~K}]=6.5$ (3 MK) with strong iron lines, up to Fe XVI. EIS observes several weak Fe XVII lines that can be used to constrain the higher temperatures. Note that all the Fe XVII lines are blended to some degree (see Del Zanna 2008 and Del Zanna \& Ishikawa 2009 for details on line identifications and atomic data). For example, the strongest Fe XVII line at $204.66 \AA$ is blended with a cool line, identified as partly due to Fe VIII at $204.704 \AA$ in Del Zanna (2009b). It turns out that the Fe XVII lines, despite having a $\log T_{\max }=6.75$, have $\log$ $T_{\text {eff }}=6.5$ in the cores of active regions, given the large DEM at 3 MK. So, effectively, the Fe XVII lines provide an upper limit to DEM values above $3 \mathrm{MK}$.

A further upper limit around $10 \mathrm{MK}$ is obtained from the Fe XXIII 263.8 A line (see Del Zanna 2008; Del Zanna et al. $2011 \mathrm{~b}$ for a discussion on blending for this line).

A number of calcium lines are often used for emission measure purposes (Warren et al. 2012), however, a number 
of problems have been found with them. Most of the Ca XIV and CaXVI lines were found to be blended. The strongest Ca XV 200.97 Å line (Del Zanna 2012b), formed around 4.5 MK, has intensities which are always at odds with those of the other lines. The CaXVII $192.82 \AA$ is the strongest line observed by EIS formed around 5-6 MK (Del Zanna 2008), but unfortunately it is severely blended. The main blending is due to the strong Fe XI $192.813 \AA$ line. Its intensity is estimated here via a branching ratio (0.21 photons) with the $188.216 \AA$ line (Del Zanna et al. 2010b). Several strong O V lines are also blended with Ca XVII (Young et al. 2007). Following our benchmark work (Del Zanna 2009a), the O v $192.9 \AA$ emission was used to estimate the contribution to the $192.8 \AA$ blend. We often found no measurable Ca XVII emission, in which case an estimate of the Ca XVII minimum intensity was considered in the DEM modelling as an upper limit.

EIS also observes several lines from Ni, Ar, S, and Si that can be used to measure quite accurately their coronal abundances (relative to iron), as described in Del Zanna (2012b) and discussed below. This is an important feature of the EIS spectra. A few weak lines from $\mathrm{K}, \mathrm{P}, \mathrm{Cr}$, and $\mathrm{Mn}$ can also provide a rough estimate for these elements, as shown below.

\subsection{Automatic DEM inversion}

The DEM spline method requires significant user interaction and is not suitable to calculate DEMs on a pixel-by-pixel basis. We have tested various inversion methods on several sets of AIA observations adopting a range of parameters. In particular, we have tested two of the most refined methods: the MCMC method of Kashyap \& Drake (1998) and the regularised inversion of Hannah \& Kontar (2012). Once the DEM was obtained, the predicted AIA count rates were then compared to the observed ones. We also predicted EIS radiances and compared them to simultaneous observations, whenever available. We found the overall results very unsatisfactory. The MCMC method failed in most places, although we note that it works relatively well when emission lines are considered (Del Zanna et al. 2011c). A similar conclusion was obtained by Testa et al. (2012a), where the MCMC method was applied to recover the results of a 3-D simulation. The Hannah \& Kontar (2012) code performed relatively well but in places failed completely to provide an acceptable representation of the observations.

Therefore, we had to develop a new method to obtain DEMs from the AIA EUV bands. As pointed out in Del Zanna et al. (2011c), part of the problem in using the AIA EUV bands lies in their broad temperature response. Another reason why inversion methods sometimes fail is related to the multiple peaks that are present in the 94, 131, 211, and $335 \AA$ bands. This problem of the multiple peaks has been partially solved with the above assumptions, i.e. by isolating the Fe XIV and Fe XVI in the 211, and $335 \AA$ bands, by removing the hot component in the $131 \AA$ band, and only using the $94 \AA$ band in the hot regions where the Fe XVIII emission can be isolated.

We chose a functional form for the DEM which allowed for the presence of near-isothermal structures, but was also multithermal and continuous. After various tests, we obtained the best results by representing the DEM in each AIA pixel as a superposition of nearly isothermal distributions, modelled as Gaussian functions of narrow width, and spaced across the entire temperature range. A similar approach was adopted by Aschwanden \& Boerner (2011) to study single loops in one AR. Typically, we used about 10 Gaussians to cover the $\log T[\mathrm{~K}]=5.6-6.6$ range, plus a few extra ones in the regions where hot emission is present. The widths of these Gaussians should be small to allow for the presence of near-isothermal plasma, but cannot be arbitrarily low, because of the limited accuracy to which the line emissivities are known (see also Judge 2010). These in turn mainly depend on the ionisation and recombination rates. After various tests, we found that a width of about 0.07 in $\log T[\mathrm{~K}]$ is a good compromise, resulting in a smooth DEM distribution.

For each pixel, a simple iterative procedure was applied by minimizing the $\chi^{2}$ by varying the heights of the Gaussians. Values with very low intensities are typically not considered for the minimization. A variant of this method has been coded to be used for the Hinode EIS spectral lines.

Any inversion code is time consuming. For a small sample of 9600 points, MCMC required 5100s, the Hannah \& Kontar (2012) $2400 \mathrm{~s}$, and the present code a similar amount, although it can be reduced with a careful selection of parameters.

If the DEM is effectively a smooth distribution, a very good approximation for it is given by

$$
\mathrm{DEM}_{\mathrm{L}}=\frac{I_{\mathrm{ob}}}{A b \int_{T} G(N, T) \mathrm{d} T}
$$

where the integral is performed over the entire temperature range where a line is formed. We use this approximation as a starting solution for the inversion, assuming for the AIA 131, 171, 193, 211, 335, $94 \AA$ channels the temperatures (log values): 5.65, $5.85,6.2,6.3,6.4,6.8$, and then interpolating them over a 0.01 temperature grid (in $\log T$ ). Note that such an approximation, as was used in Widing \& Feldman (1989) and subsequent papers to estimate relative elemental abundances, fails completely when the plasma is nearly isothermal (Del Zanna et al. 2001, 2003; Del Zanna 2003b).

An upper limit to the DEM distribution is easily obtained by considering the emission measure loci. The loci of the curves $I_{\mathrm{ob}} /(A b \times G(N, T))$ represent, at each temperature, the upper limit to the value of $E M_{h}(T, \Delta T)$. The limit is obtained only if the plasma is strictly isothermal at that temperature. The EM Loci method was applied for the first time by Strong (1978) and Veck et al. (1984) to the analysis of solar X-ray flare spectra, and later by Del Zanna \& Mason (2003) to SOHO CDS observations of solar active regions. The method has since become very common in the literature.

The upper boundary for the $E M$ has been converted to an upper boundary for the DEM distribution by assuming a constant DEM in each temperature bin. As a lower boundary, the $\mathrm{DEM}_{\mathrm{L}}$ values reduced by two orders of magnitude were chosen.

We have calculated the instrument responses $G(N, T)$ in the $T[\mathrm{~K}]=5-7.5$ range, but have limited the inversion to a more restricted temperature range, depending on the type of observations considered. The results of the inversion are quite sensitive to this choice. The DEM values in the $\log T[\mathrm{~K}]=5-5.5$ range are unconstrained by AIA, so we have adopted typical values in the range $10^{20.7-20.0} \mathrm{~cm}^{-5} \mathrm{~K}^{-1}$ for on-disk observations.

\section{Validating the DEM method with AIA and EIS simultaneous observations}

We have tested our new DEM method on a few AIA and EIS simultaneous observations of active regions. We provide here two examples. Figure 4 shows the result of the DEM modelling for the on-disk AR observation of 2010 Oct. 26 obtained from the AIA data. The agreement between observed and estimated 

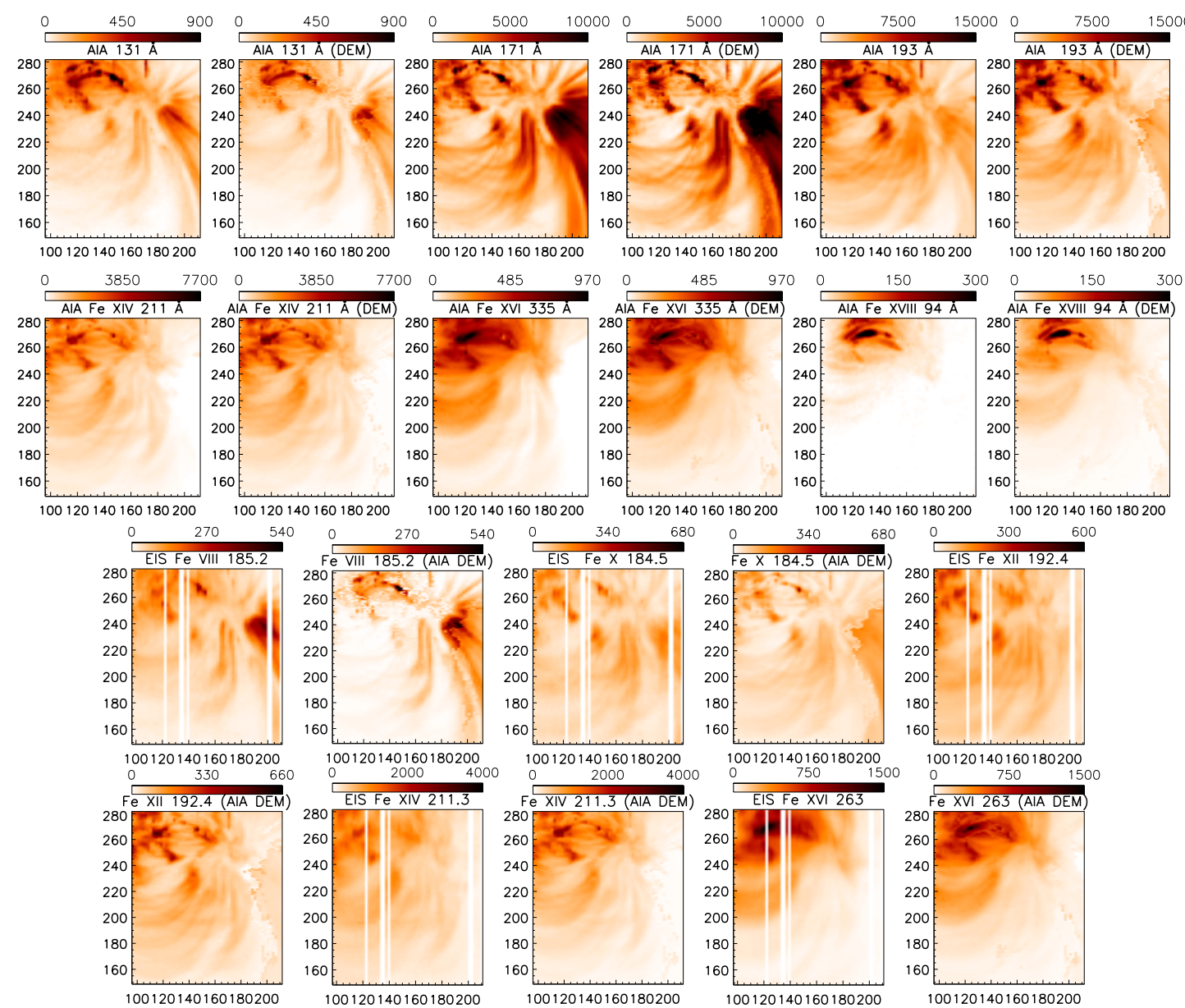

Fig. 4. Top two rows: observed and estimated (from the AIA DEM modelling) AIA images (negative, DN/s) of the on-disk AR observation of 2010 Oct. 26, rebinned to the EIS resolution. Lower two rows: monochromatic images (negative, photons $\mathrm{cm}^{-2} \mathrm{~s}^{-1} \operatorname{arcsec}^{-2}$ ) in EIS lines, observed and estimated from the AIA DEM modelling.

AIA count rates is truly remarkable. The DEM was also used to predict the Hinode EIS radiances (in photons $\mathrm{cm}^{-2} \mathrm{~s}^{-1} \operatorname{arcsec}^{-2}$ ), which are compared to the observed ones in the same figure. The agreement is surprisingly good, both in terms of absolute and relative values. The upper part of the FOV was difficult to model, because hot emission from Fe XVIII is present. The results for the on-disk AR observation of 2010 Nov. 23 are shown in Fig. 5. The lower part of the FOV was particularly difficult to fit because the hotter lines are very weak, while the cooler ones are very strong. This is a region where other methods we have tested typically failed.

In both cases, we adopted the coronal abundances that are discussed below. The emissivities were calculated with the present atomic data (listed in the beginning of Sect. 3) and a constant electron density of $2 \times 10^{9} \mathrm{~cm}^{-3}$, a typical average $A R$ value. The excellent agreement confirms the validity of the AIA and EIS calibrations, as well as the accuracy of the atomic data. The comparisons also indicate a good reliability of the procedure, which provides confidence in the results that are obtained with the full-resolution AIA data, described below.

Obviously, the fact that in most pixels the method is able to reproduce AIA count rates and EIS radiances within about $30 \%$ does not necessarily mean that the obtained DEM is an exact solution. Indeed, as mentioned, the inversion method is ill-posed and there is no unique solution. Also, broad-band imaging can never replace the accuracy and amount of information that can be obtained with spectroscopic measurements.

\section{The main contributions to the AIA EUV bands in the cores of ARs}

To assess the various contributions to the AIA bands, a large region within the core of the AR observed on the 2010 Oct. 26 was selected (see Fig. 4), also to avoid possible small misalignments between EIS and AIA, and to increase the signal-to-noise ratio $(\mathrm{S} / \mathrm{N})$ in the EIS spectra. Table 1 shows the observed AIA count rates, together with the predicted ones for the 193 and $211 \AA$ bands, obtained by convolving the calibrated EIS spectra with the AIA (ground calibration) effective areas. Good agreement is found. A large uncertainty in the AIA DN estimated directly from EIS is related to the measurement of the EIS bias, done here by taking average values around 205.5, and $210.5 \AA$ in regions free of strong lines, for the 193 and the $211 \AA$ bands. Good agreement is found. The EIS $171 \AA$ line is very uncertain $(50 \%)$ because it is at the limit of the detector and has very low counts.

Figure 6 (top) shows the DEM obtained with the spline method and the elemental abundances discussed below. We used this DEM to calculate line and continuum emission for all the AIA bands and calculate the total AIA simulated count rates, displayed in Table 1. Very good agreement is found.

In the $171 \AA$ band, the main Fe IX line contributes $1930 \mathrm{DN} / \mathrm{s}$, while about 10\% (310 DN/s) comes from Ni XIV $171.37 \AA$. In the $193 \AA$ band, about half comes from the Fe XII lines. In the $211 \AA$ band, the Fe XIV $211.3 \AA$ line 

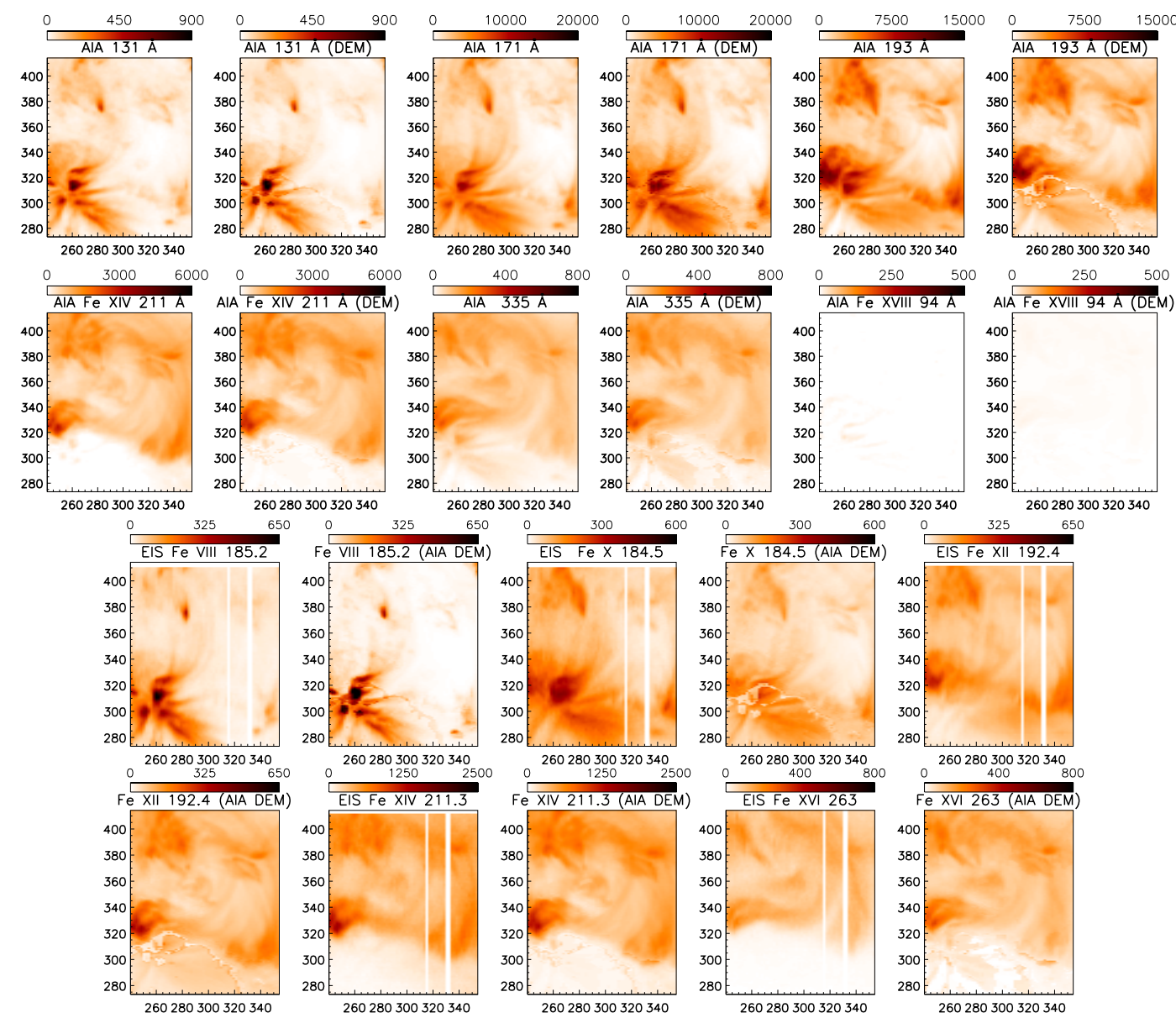

Fig. 5. Top two rows: observed and estimated (from the AIA DEM modelling) AIA images (negative, DN/s) of the on-disk AR observation of 2010 Nov. 23, rebinned to the EIS resolution. Lower two rows: monochromatic images (negative, photons $\mathrm{cm}^{-2} \mathrm{~s}^{-1} \operatorname{arcsec}^{-2}$ ) in EIS lines, observed and estimated from the AIA DEM modelling.

Table 1. Observed and predicted (from the EIS data and the DEM modelling) SDO/AIA count rates (averaged DN/s per EIS pixel) for the 2010 Oct. 26 (on-disk) region.

\begin{tabular}{lccc}
\hline \hline $\begin{array}{l}\text { Band } \\
(\AA)\end{array}$ & $\begin{array}{c}\text { Obs. } \\
(\text { AIA })\end{array}$ & $\begin{array}{c}\text { Pred. } \\
(\text { EIS })\end{array}$ & $\begin{array}{c}\text { Pred. } \\
(\text { DEM })\end{array}$ \\
\hline 94 & 45 & - & $45(0 \%)$ \\
131 & 169 & - & $127(-25 \%)$ \\
171 & 2385 & - & $2790(17 \%)$ \\
193 & 4913 & $5570(13 \%)$ & $4694(-4 \%)$ \\
211 & 2610 & $2382(-9 \%)$ & $3385(30 \%)$ \\
335 & 339 & - & $368(10 \%)$ \\
\hline
\end{tabular}

contributes about $50 \%$ of the predicted counts. In the $335 \AA$ band, the Fe XVI $335.4 \AA$ line contributes about $80 \%$.

A significant fraction of the counts in the 94 and $131 \AA$ bands comes from the free-bound continuum, formed around $3 \mathrm{MK}$. With the present abundances, the continuum contributes about $10 \%$ and $36 \%$, respectively. With photospheric abundances, the contributions increase to about $20 \%$ and $50 \%$ for the 94 and $131 \AA$ bands.

\subsection{The AIA $94 \AA$ band and FeXVIII}

The AIA simulated count rates as a function of temperature and wavelength are shown in Fig. 6. A significant contribution comes from Fe XVIII. After Fe XVIII, the newlyidentified Fe XIV 93.61 $\AA$ line produces the main contribution to the band, while minor contributions come from Fe X, and the set of lines from Fe IX and Fe XII discussed previously.

It is interesting to note that the Fe XVIII intensity is due to the 3 MK plasma, as Fig. 6 (middle) shows. This is caused by the large DEM peak at $3 \mathrm{MK}$, a temperature where the Fe XVIII contribution function is only about $8 \%$ its peak value at $7 \mathrm{MK}$. This is a surprising result, although it somewhat uncertain because it relies on the accuracy in the ion abundance towards the wings. Note that in contrast the CaXVII $192.8 \AA$ line has a predicted emissivity at $3 \mathrm{MK}$ of only $1 \%$ its peak value at $6 \mathrm{MK}$. This is consistent with the fact that we found several cases where this line is not observed when in contrast Fe XVIII is expected to be present. This is another somewhat surprising result, considering that CaXVII has a lower peak formation temperature (6 MK) than Fe XVIII (7 MK).

Obtaining the Fe XVIII emission with DEM modelling based on EIS lines is clearly difficult, especially when Fe XVII lines are not measurable. A better way to estimate the Fe XVIII contribution to the AIA $94 \AA$ band is to subtract all the known contributions from the observed count rates. The Fe VIII-Fe XIV contributions can in fact be estimated quite accurately using the EIS observations. We applied our automatic DEM inversion for each EIS pixel using 12 iron lines. We then calculated the contributions of the various ions and the continuum for each EIS pixel, in AIA $94 \AA \mathrm{DN} / \mathrm{s}$. The results for the two selected ARs 
G. Del Zanna: The multi-thermal emission in solar active regions
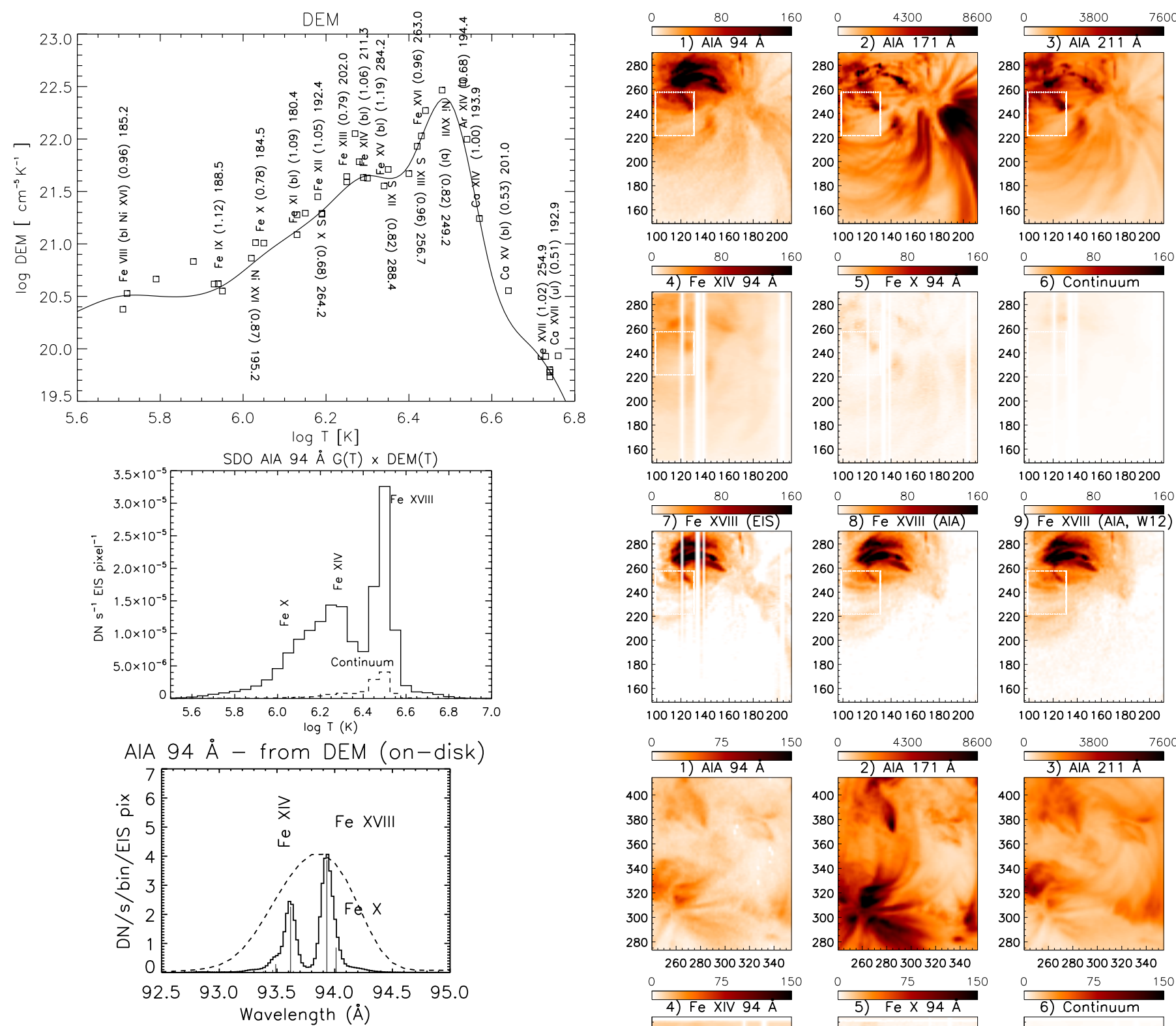

Fig. 6. Above: DEM of the 2010 Oct. 26 active region core. The numbers in parentheses are the ratios between predicted and observed intensities. The points are plotted at the temperature $T_{\max }$, and at the theoretical vs. the observed intensity ratio multiplied by the DEM value. Below: corresponding AIA $94 \AA$ count rates as a function of temperature and wavelength (the dashed curve is the normalised AIA effective area).

are plotted in Fig. 7. Panels 7 in the same figure show the observed count rates with all the various known contributions subtracted. The residual emission is expected to be due mostly to Fe XVIII, although at least one relatively strong emission line is still unidentified. The lower panel (Nov. 23 observation) indicates that the unidentified line has a low formation temperature, close to Fe VIII.

It is clear that the newly-identified Fe XIV 93.61 $\AA$ always provides an important contribution to the $94 \AA$ band, the dominant one whenever Fe XVIII emission is not present. A significant contribution also comes from the continuum, while a minor one from Fe X 94.012 ̊. The Fe VIII, Fe IX, Fe XII lines produce a negligible contribution. This is a new and important result. There is good agreement between the present and the
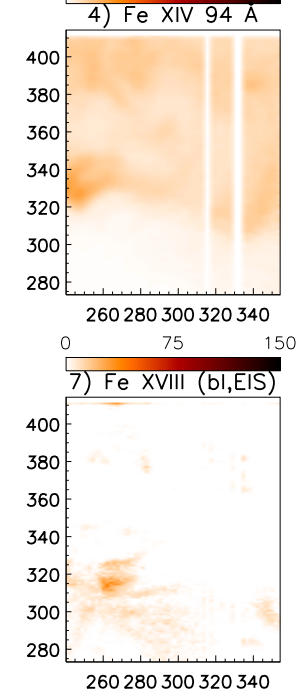
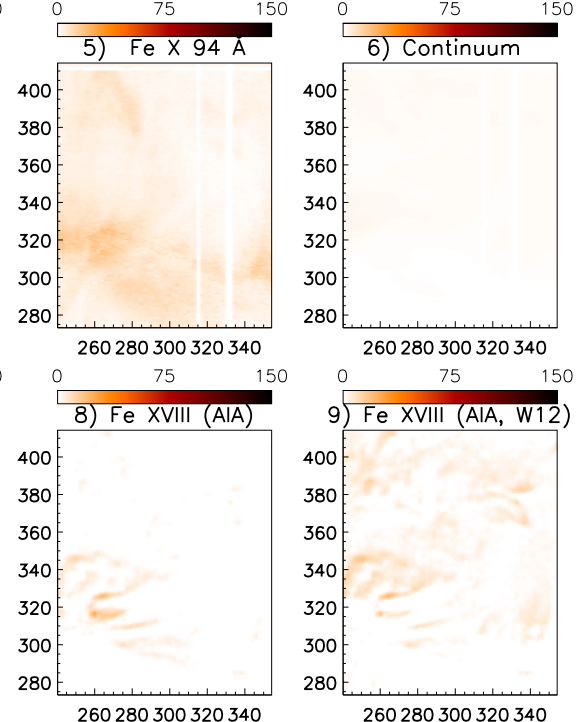

Fig. 7. AIA images (negative, DN/s/pixel) of the on-disk AR observations of 2010 Oct. 26 (above) and Nov. 23 (below), rebinned onto the EIS resolution. Panels 4, 5, 6 show the $94 \AA$ A predicted count rates in Fe XIV, Fe X, and the continuum. Panels 7 and 8 show the residual count rates in the AIA $94 \AA$ (due to Fe XVIII), estimated from EIS and AIA. Panels 9 show the Fe XVIII estimate of Warren et al. (2012). 

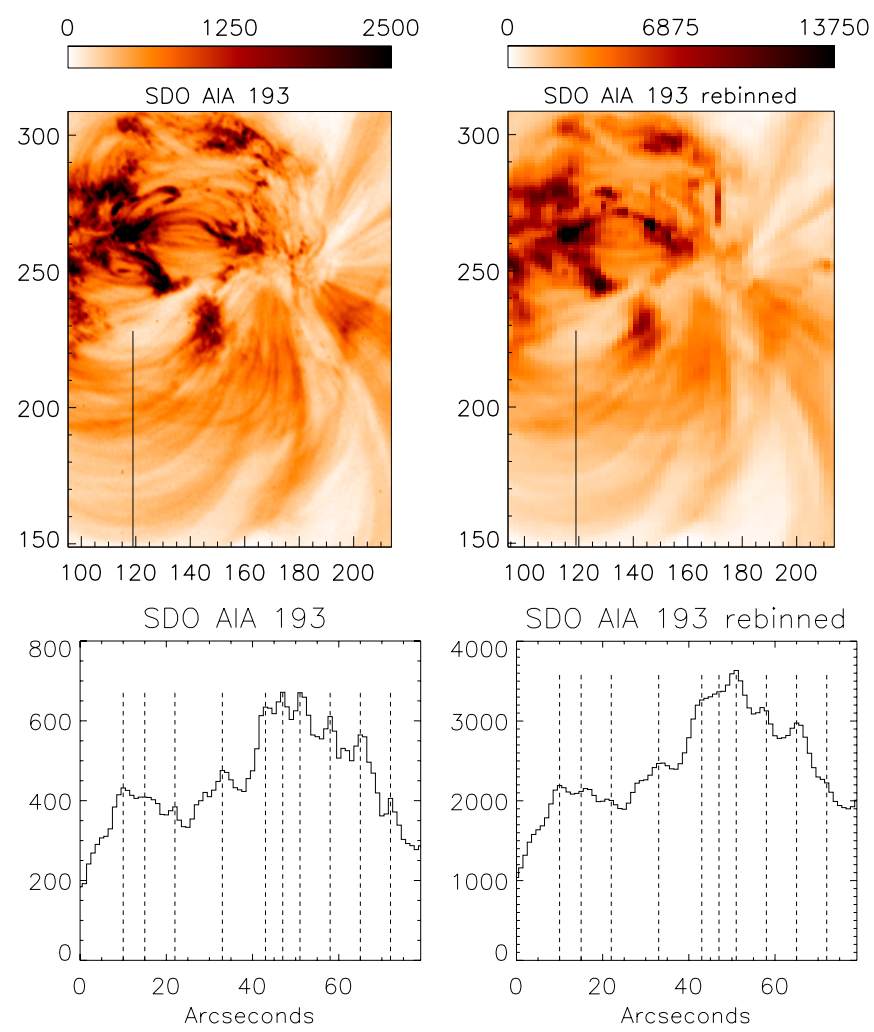

Fig. 8. Above: zoom up of a full-resolution AIA $193 \AA$ Amage (left, negative, at 11:40 UT) of the 2010 Oct. 26 active region within the EIS FOV, and the corresponding rebinned image obtained by converting the AIA observations into the EIS spatial and temporal resolution (right, negative). Below: profiles of the count rates along the N-S segment shown in the images. The locations of a few loop structures that appear as nearly resolved in the full-resolution AIA $193 \AA$ image are indicated with dashed lines.

Warren et al. (2012) method to estimate the Fe XVIII emission, as shown in Fig. 7.

\section{The multi-thermal emission at the AIA resolution}

One of the aims of this paper is to discuss the multi-thermal emission in the AR cores at the higher AIA resolution. To illustrate how significant the increase is, we consider the 2010 Oct. 26 AR observations. Hinode EIS observed the AR during 10:50-11:50 UT. We compare in Fig. 8 a full-resolution AIA $193 \AA$ image taken at 11:40 UT with the rebinned image, obtained by converting the AIA observations into the EIS spatial and temporal resolution (the same as was shown previously in Fig. 4). We corrected for the AIA stray light using the results of Poduval et al. (2013). We note that the correction for the stray light is significant (up to $50 \%$ changes) for some active regions and enhances the contrast between the loop structures and the background.

Figure 8 also shows profiles of the count rates along a segment shown in the images. Several loop structures appear almost resolved by AIA (they are indicated with dashed lines), but many of them become hardly visible at the EIS resolution. We note that there is a region free of loop structures, near the AR core (at pixel position 80), where the AIA count rates are similar to those far away from the AR (at pixel position 0 , the bottom of the image). In the rebinned image, the count rates in this core region are instead almost twice the values at the bottom of the image. This clearly illustrates that a factor of two increase in the "unresolved" background observed by Hinode EIS (and SOHO CDS) in AR cores can simply be caused by its lower spatial resolution.

We then performed the automatic AIA DEM modelling on the full-resolution AIA data of the active region using the set of abundances discussed below. To increase the $\mathrm{S} / \mathrm{N}$, we averaged the AIA images over a minute, between 11:40 and 11:41 UT. We corrected for the AIA stray light using the results of Poduval et al. (2013) and applied our corrections for the AIA plate scale. We then calculated the predicted radiances in a sample of EIS lines. Sample results are shown in Fig. 9, centred on a bundle of 3 MK loops, clearly seen in AIA $335 \AA$ (Fe XVI). These images, together with the intensity profiles across the loops, clearly show the presence of a multitude of loops that are mostly not co-spatial at the various temperatures, and become less well resolved at higher temperatures. The locations of a few cool loops which appear as almost resolved in Fe VIII and Fe IX are shown in the profiles of Fig. 9 as dashed lines. Higher-temperatures loops in e.g. Fe XII are normally not co-spatial with the cooler emission. In each single AIA pixel, there is clearly some superposition of different structures along the line of sight.

The "contrast" of the structures as seen in single emission lines is clearly enhanced, compared to the original AIA images, which confirms that the multi-thermal nature of the AIA bands is somewhat "blurring" our view of the AR structures. For example, we notice the significant differences between the AIA $131 \AA$ image and the reconstructed Fe VIII towards the AR core (upper images, 100-150" in the profiles). The DEM modelling predicts very little unresolved background emission in the AR core in lines formed up to $1 \mathrm{MK}$ (as Fe X). The unresolved background emission is instead about a factor of 34 higher in Fe XII- Fe XIII, and much higher in Fe XIV-Fe XVI, compared to the values outside the core (in the bottom of the image). This confirms the TRACE and SOHO CDS results of Del Zanna \& Mason (2003), although the higher AIA resolution now shows much lower levels of unresolved emission, relative to the total emission (less than $50 \%$ instead of $80 \%$ ). The increased spatial resolution clearly allows us to resolve more and more structures and enables us to better "see through" them. One question naturally arises: what is this unresolved emission? It is likely composed of a multitude of loops of sizes well below the resolution of current instrumentation. We believe that a factor of 2-3 increase is just due to an increase in the iron abundance, rather than a density effect, as discussed below.

There is an unresolved 3 MK bundle of loops seen in Fe XVI, with footpoints approximately aligned along the E-W direction. The bundle of 3 MK loops is also clearly visible with EIS (cf. Fig. 4). We note that these loops are also clearly visible in Fe XIV, although most of the emission in this ion is formed at $3 \mathrm{MK}$ and not at its peak formation temperature of $2 \mathrm{MK}$, as discussed below.

\section{Elemental abundances and path lengths for the 3 MK loops}

A region within the centre of this $3 \mathrm{MK}$ bundle of loops (at coordinates 110, 210, see Fig. 9) with no underlying obvious emission, from e.g. moss regions, was chosen for detail analysis using the Hinode EIS spectral lines. We obtained averaged EIS calibrated spectra, and measured the average electron density from the Fe XIV lines and found $10^{9.2} \mathrm{~cm}^{-3}$. We note that the Fe XIV lines are not formed at peak ion abundance, so the densities need to be obtained by calculating the theoretical ratios 

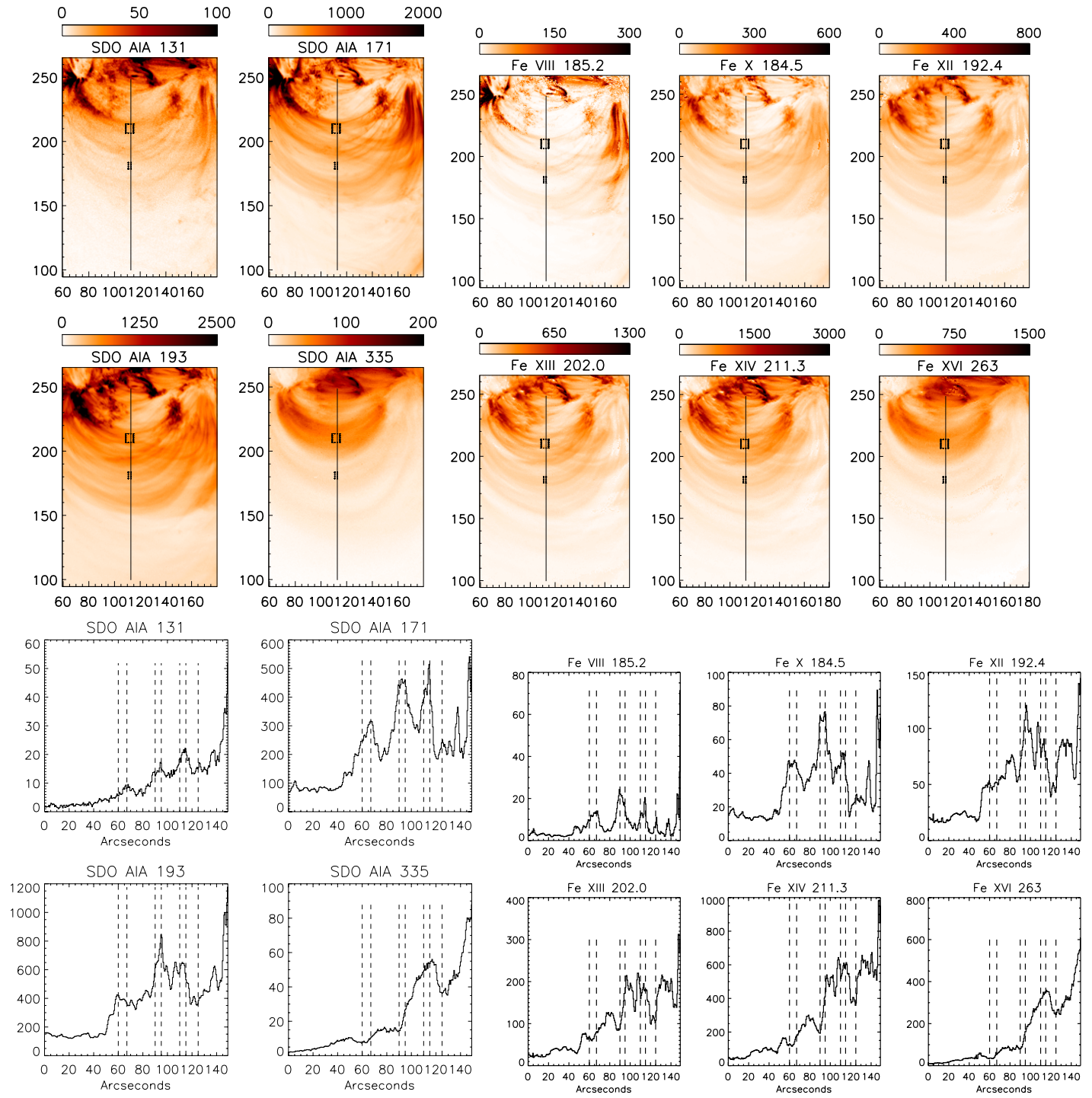

Fig. 9. Top left: full-resolution AIA images of the 2010 Oct. 26 active region. The two selected regions are shown (the larger on the 3 MK loops, the smaller for the background), as well as a cut across the 3 MK loops. Top right: the corresponding radiances in a few EIS lines, predicted from the AIA DEM modelling. Below: profiles of the count rates and radiances across the 3 MK loops.

at $3 \mathrm{MK}$, something that is not normally considered in the literature. We obtained consistent results from other ions.

We performed a DEM modelling using the EIS iron lines, adopting at first the photospheric abundances of Asplund et al. (2009). We then calculated the path length as described in Sect. 4, by integrating the DEM in the $\log T[\mathrm{~K}]=6.3-6.6$ range and considering a density of $10^{9.2} \mathrm{~cm}^{-3}$. We obtained $350^{\prime \prime}$, an unreasonable number, considering that the size of the bundle of loops as seen in the plane of the sky is only of the order of $20^{\prime \prime}$ (see e.g. Fig. 9). Considering that the EIS calibration is now quite well established (Del Zanna 2013), and the atomic data for the iron lines are quite accurate now, there are only two improvements that can be applied to reduce the path length. The first one regards "background/foreground" subtraction. Clearly, the $3 \mathrm{MK}$ bundle of loops is embedded in an environment rich of loops at lower temperatures, plus some line-of-sight emission at $3 \mathrm{MK}$. Subtracting this emission lowers the observed emission measure. Given the EIS resolution and FOV, there is a limited choice, so a small nearby region (where Fe XVI emission is low) was chosen (at coordinates 110, 180, see Fig. 9). Subtracting the emission from this region not only reduces the emission measure, but also increases the averaged density (which also reduces the path length), to a value of about $10^{9.5} \mathrm{~cm}^{-3}$, providing a path length of $54^{\prime \prime}$.

The electron density was obtained using the "emissivity ratio" technique (see Del Zanna et al. 2004 for details), which divides the observed intensity of a line $\left(I_{\mathrm{ob}}\right)$ by its emissivity:

$F_{j i}=\frac{I_{\mathrm{ob}} N_{\mathrm{e}} C}{N_{j}\left(N_{\mathrm{e}}, T_{\mathrm{e}}\right) A_{j i}}$

calculated at a fixed electron temperature $T_{\mathrm{e}}$ (at $3 \mathrm{MK}$ ) and plotted as a function of the density $N_{\mathrm{e}}$ ( $C$ is a scaling constant). $N_{j}$ is the population of the upper level, while $A_{j i}$ is the transition probability of spontaneous emission. Only Fe XIV and S XI provide useful diagnostics because the lines are strong in a $3 \mathrm{MK}$ plasma. The two ions provide similar densities, as shown in Fig. 10. There are other density-sensitive Ni XV and Ca XV lines but they are weak, confirming that the density is not higher than $10^{9.5} \mathrm{~cm}^{-3}$.

The reduction in the path length is significant but not enough, so reasonable path lengths are obtained only by increasing the iron abundance. We adopted a reasonable increase of $0.5 \mathrm{dex}$ 

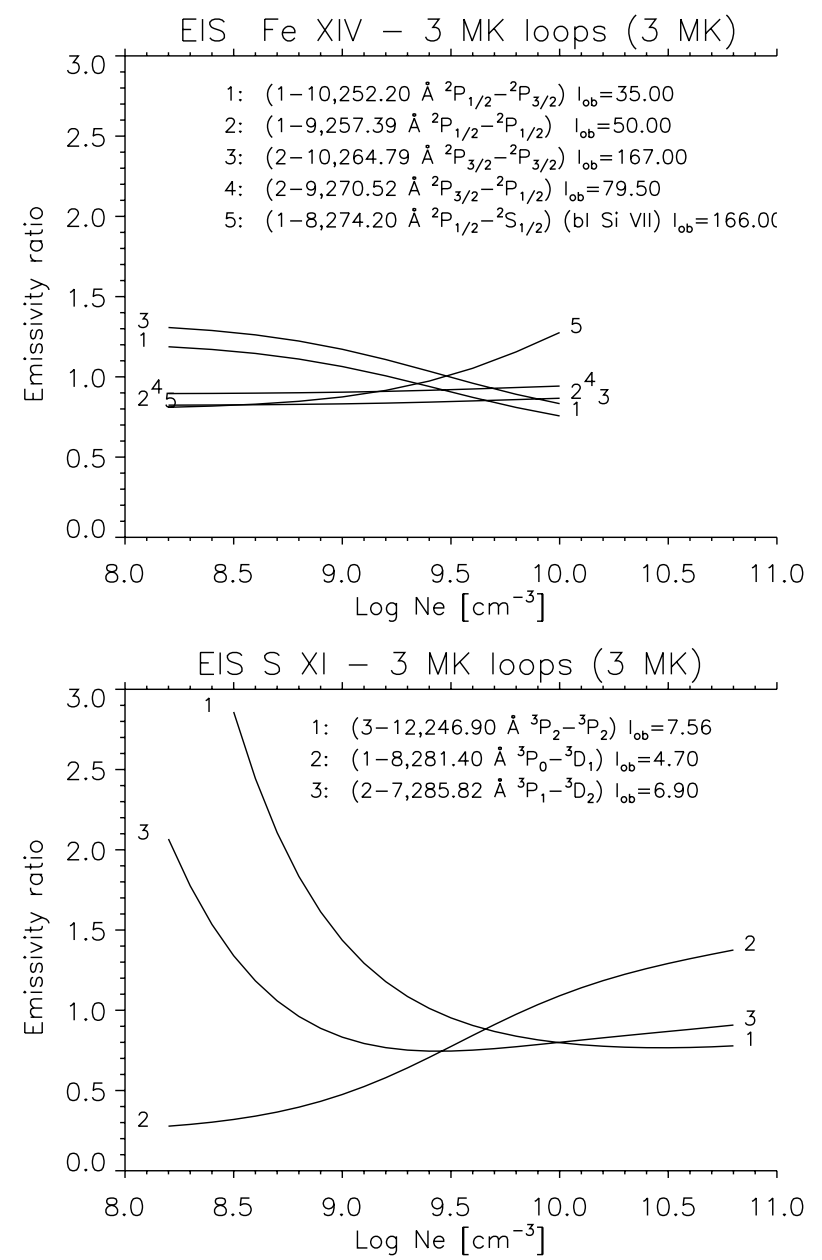

Fig. 10. Emissivity ratio curves relative to the main Fe XIV and S XI transitions observed by Hinode EIS on the bundle of 3 MK loops of Fig. 9.

(a factor of 3.16 increase), providing a path length of $17^{\prime \prime}$. This is a reasonable value, close to the overall size of the bundle of loops, and that implies a spectroscopic filling factor close to one. We note that the factor of 3.16 increase is consistent with typical X-ray measurements of the relative $\mathrm{O} / \mathrm{Fe}$ and $\mathrm{Ne} / \mathrm{Fe}$ ratios (which will be discussed in a separate paper), assuming that the high-FIP elements have the photospheric (Asplund et al. 2009) abundances.

Having fixed the iron abundance to 8.0 dex (on the usual logarithmic scale where hydrogen is 12), we then used the DEM modelling to find the abundances shown in Table 2. Fig. 11 shows the DEM obtained with the spline method from the background-subtracted intensities. For comparisons, it also shows the DEM obtained from the same set of abundances but without subtracting the background. The DEM is very well constrained by strong lines from all the iron ionisation stages from Fe VIII to Fe XVI. The Fe XVII lines provide a constrain at higher temperatures. The DEM is nearly isothermal, centred at $3 \mathrm{MK}$, and with a peak value two orders of magnitude higher than the emission measures at lower or higher temperatures.

Table 3 summarises the results for the strongest lines. Excellent agreement between theory and observations is found, although we recall that discrepancies of factors of two are present if the EIS ground calibration is adopted. The abundances of $\mathrm{K}, \mathrm{Ar}, \mathrm{Ca}, \mathrm{P}, \mathrm{S}$, and $\mathrm{Ni}$ (relative to iron) are well constrained, since the KXIII, Ar XIII, ArXIV, CaXIV, P XII, S XII S XIII, Ni XV, Ni XVI, and Ni XVII lines are all formed around $3 \mathrm{MK}$
Table 2. Present hot core loops abundances.

\begin{tabular}{lcccc}
\hline \hline El. & Phot. & Coronal & Present & FIP $(\mathrm{eV})$ \\
\hline $\mathrm{H}$ & 12 & 12 & 12 & 13.56 \\
$\mathrm{C}$ & 8.43 & 8.59 & - & 11.26 \\
$\mathrm{~N}$ & 7.83 & 8.0 & - & 14.53 \\
$\mathrm{O}$ & 8.69 & 8.89 & - & 13.61 \\
$\mathrm{Ne}$ & $7.93^{*}$ & 8.08 & - & 21.56 \\
$\mathrm{Mg}$ & 7.6 & 8.15 & 8.10 & 7.64 \\
$\mathrm{Al}$ & 6.45 & 7.04 & 6.95 & 5.98 \\
$\mathrm{Si}$ & 7.51 & 8.10 & 8.00 & 8.15 \\
$\mathrm{P}$ & 5.41 & - & 5.41 & 10.49 \\
$\mathrm{~S}$ & 7.12 & 7.27 & 7.17 & 10.36 \\
$\mathrm{Ar}$ & $6.40^{*}$ & 6.58 & 6.30 & 15.76 \\
$\mathrm{~K}$ & 5.03 & - & 5.53 & 4.34 \\
$\mathrm{Ca}$ & 6.34 & 6.93 & 6.87 & 6.11 \\
$\mathrm{Cr}$ & 5.64 & - & 6.14 & 6.78 \\
$\mathrm{Mn}$ & 5.43 & - & 5.93 & 7.43 \\
$\mathrm{Fe}$ & 7.50 & 8.10 & 8.00 & 7.87 \\
$\mathrm{Ni}$ & 6.22 & 6.84 & 6.53 & 7.63 \\
\hline
\end{tabular}

Notes. Photospheric (Phot) abundances are from Asplund et al. (2009), the coronal ones from Feldman et al. (1992). Ar and $\mathrm{Ne}$ do not have photospheric measurements.

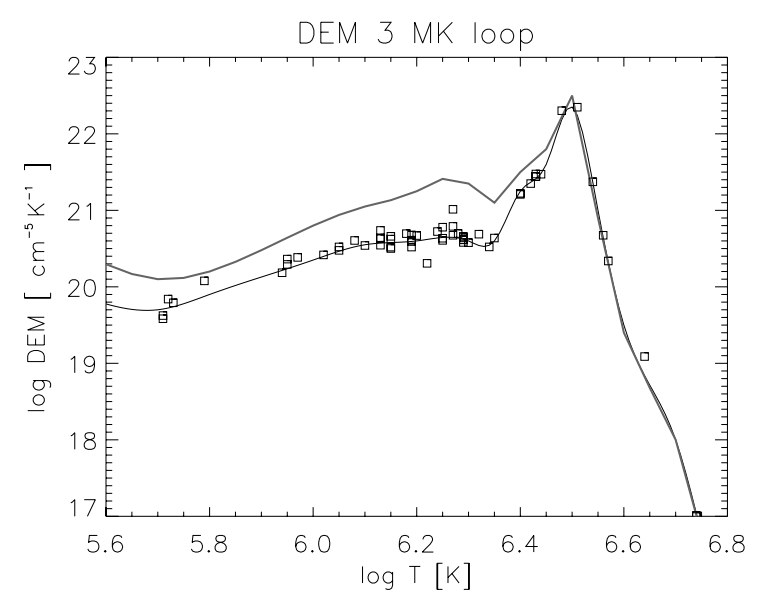

Fig. 11. DEM of the 2010 Oct. 26 active region 3 MK loops, obtained from the background-subtracted intensities, with the present coronal abundances. The points are plotted at the temperature $T_{\max }$, and at the theoretical vs. the observed intensity ratio multiplied by the DEM value. The grey thick line shows the DEM obtained directly from the EIS intensities without any background subtraction.

(see the effective temperatures in Table 3). We recommend that the following lines are used for future studies: K XIII 208.109 $\AA$, ArXIV $194.396 \AA$, CaXIV 193.866 A, P XII $278.286 \AA$, S XII $288.421 \AA$, S XIII $256.685 \AA$, Ni XV $176.710 \AA$, Ni XVI 195.271 A, Ni XVII 249.185 A. The P XII $278.286 \AA$ is weak but is the resonance line for this ion, so effectively the EIS measurement provides an upper limit to the $\mathrm{P}$ abundance. A similar argument applies to the K XIII 208.109 A resonance line and the Cr IX 210.611 $\AA$ line. The strong Ca XV 200.971 $\AA$ is at odds with all the other ones, indicating possible problems in the calcium ion abundances or with the atomic data for this ion, or the presence of an unknown blend.

The lower-temperature Ar XI is at odds with the other ions. This could partly be due to different abundances in the lowertemperature plasma, as the SX abundance seem to suggest as well. The present abundances differ significantly from previous 
Table 3. Observed and predicted radiances for the 3 MK loops.

\begin{tabular}{|c|c|c|c|c|c|c|c|}
\hline$\lambda_{\mathrm{obs}}$ & $I_{\mathrm{obs}}$ & $T_{\max }$ & $T_{\text {eff }}$ & $R$ & Ion & $\lambda_{\exp }$ & $r$ \\
\hline 176.69 & 18.9 & 40 & 6.47 & 1.06 & Nix & 76.710 & 0. \\
\hline & 40.8 & & 11 & 0.97 & $\mathrm{Fe} \mathrm{X}$ & & 1.00 \\
\hline 180.39 & 62.9 & .13 & 6.17 & 1.06 & Fe XI & 01 & 0.99 \\
\hline \multirow[t]{2}{*}{183.45} & 9.1 & 6.56 & 6.50 & 0.94 & Ar XIV & 183. & 0.13 \\
\hline & & & & & Ca XIV & 183.460 & 0.87 \\
\hline 184.53 & 19.9 & 6.05 & 6.13 & 0.88 & $\mathrm{Fe} X$ & 184.5 & 0.94 \\
\hline \multirow[t]{2}{*}{185.22} & 40.7 & 5.73 & 6.39 & 0.89 & Ni XVI & 30 & 0.74 \\
\hline & & & & & Fe VIII & & 0.25 \\
\hline \multirow[t]{2}{*}{185.66} & 2.1 & 6.40 & 6.46 & 1.10 & $\mathrm{Ni} 2$ & & 0.88 \\
\hline & & & & & $\mathrm{Ni} X \mathrm{~V}$ & & 0.06 \\
\hline \multirow[t]{2}{*}{186.61} & 27.4 & 5.72 & 6.39 & 0.76 & Ca XIV & & 0.70 \\
\hline & & & & & $\mathrm{Fe} \mathrm{V}$ & & 0.29 \\
\hline 188.21 & 40.9 & 6.13 & 6.17 & 0.86 & Fe XI & & 1.00 \\
\hline \multirow[t]{3}{*}{188.49} & & 5.95 & 6.06 & 0.88 & Fe XII & & 0.08 \\
\hline & & & & & Mn IX & & 0.06 \\
\hline & & & & & Fe IX & 188. & 0.83 \\
\hline 188.80 & 6.0 & 6.27 & 6.44 & 0.43 & Ar XI & 188.8 & 0.97 \\
\hline \multirow[t]{2}{*}{189.93} & 3.7 & 5.94 & 6.06 & 1.07 & Fe XII & 189 & 0.09 \\
\hline & & & & & Fe IX & & 0.89 \\
\hline \multirow[t]{2}{*}{191.40} & 4.5 & 6.51 & 6.48 & 0.79 & Ar XIV & 191. & 0.62 \\
\hline & & & & & Fe XV & & 0.34 \\
\hline 192.39 & 21.4 & 6.19 & 6.25 & 0.96 & Fe XII & 192 & 1.00 \\
\hline & & & 6.16 & 0.88 & $\mathrm{Fe} X \mathrm{XI}$ & & 0.95 \\
\hline 193.87 & 23.2 & & 6.50 & 0.96 & Ca XIV & & 1.00 \\
\hline \multirow[t]{2}{*}{194.05} & 3.2 & 6.19 & 6.46 & 1.18 & $\mathrm{Ni}$ & & 0.88 \\
\hline & & & & & & & \\
\hline & & & & & & & 0.99 \\
\hline & 6. & & & 1.00 & & & 0.99 \\
\hline & 7. & & 6.4 & 0.95 & Ni XVI & & 0.88 \\
\hline 5.54 & 1. & 6 & 6.46 & 0.83 & Ni XV & & 0.93 \\
\hline 200.99 & 14.7 & 6.64 & 6.50 & 0.56 & $\mathrm{CaXV}$ & 200.971 & 0.98 \\
\hline 202.04 & 37.9 & 6.25 & 6.36 & 1.09 & Fe XIII & 202.044 & 1.00 \\
\hline 204.65 & 5.2 & .74 & 6.50 & 1.05 & Fe XVII & 204.668 & 0.99 \\
\hline \multirow[t]{2}{*}{205.92} & 0.936 & 5.46 & 6.45 & 0.87 & Ar XIII & 205 & 0.81 \\
\hline & & & & & XIII & & 0.07 \\
\hline \multirow[t]{2}{*}{208.12} & 4.0 & 5.50 & 6.48 & 0.51 & K XIII & 208 & 0.90 \\
\hline & & & & & & & 0.07 \\
\hline \multirow[t]{2}{*}{208.31} & 4.6 & 6.19 & 6.43 & 0.82 & $\mathrm{CaXV}$ & 21 & 0.65 \\
\hline & & & & & & & 0.33 \\
\hline \multirow[t]{3}{*}{208.63} & 4.9 & 6.24 & 6.43 & 0.82 & CaXVI & & 0.51 \\
\hline & & & & & & & 0.39 \\
\hline & & & & & & & 0.05 \\
\hline 1 & 13.0 & 6.30 & 6.44 & 1.05 & & & 1.00 \\
\hline & 19.1 & & 6.35 & 1.02 & Fe XIII & & 0.99 \\
\hline 246.90 & 7.6 & 27 & 6.42 & 0.72 & & 95 & 1.00 \\
\hline 249.17 & 199.0 & .48 & 6.49 & 0.76 & Ni XVII & 249.185 & 1.00 \\
\hline 251.06 & 137.0 & 6.43 & 6.49 & 0.86 & Fe XVI & 251.063 & 1.00 \\
\hline 251.94 & 51.3 & 6.25 & 6.35 & 0.73 & Fe XIII & 251.952 & 0.99 \\
\hline 252.19 & 35.4 & 6.29 & 6.44 & 1.02 & Fe XIV & 252.199 & 1.00 \\
\hline 254.87 & 5.7 & 6.74 & 6.49 & 1.09 & Fe XVII & 254.885 & 0.94 \\
\hline 256.67 & 200.0 & 6.42 & 6.48 & 1.04 & S XIII & 85 & 1.00 \\
\hline \multirow[t]{2}{*}{257.38} & 50.4 & 6.29 & 6.44 & 1.11 & Fe XIV & 257.394 & 0.99 \\
\hline & & & & & Fe XIII & 254.905 & 0.06 \\
\hline & & 6.15 & 6.25 & 1.20 & Si X & 261.056 & 1.00 \\
\hline 262.98 & 225.0 & 6.43 & 6.49 & 0.93 & Fe XVI & 262.976 & 1.00 \\
\hline 64.23 & 8.7 & 6.18 & 6.31 & 0.7 & S X & 264.230 & 0.98 \\
\hline & 167.0 & 6.29 & 6.43 & 0.96 & Fe XIV & 264.7 & 0.98 \\
\hline 265.00 & 21.9 & 6.43 & 6.49 & 0.93 & Fe XVI & 265.000 & 1.00 \\
\hline
\end{tabular}

Notes. $\lambda_{\mathrm{obs}}(\AA)$ are the observed wavelengths, $I_{\mathrm{obs}}$ the backgroundsubtracted Hinode EIS radiances (photons $\mathrm{cm}^{-2} \mathrm{~s}^{-1} \operatorname{arcsec}^{-2}$ ) obtained with our new EIS calibration (Del Zanna 2013), $T_{\max }$ and $T_{\text {eff }}$ the maximum and effective temperature (log values, in $\mathrm{K}$; see text), $R$ the ratio between the predicted and observed radiances, Ion and $\lambda_{\exp }(\AA)$ the main contributing ion, and $r$ the fractional contribution to the blend.
Table 3. continued.

\begin{tabular}{lccccccc}
\hline \hline$\lambda_{\text {obs }}$ & $I_{\text {obs }}$ & $T_{\max }$ & $T_{\text {eff }}$ & $R$ & Ion & $\lambda_{\exp }$ & $r$ \\
\hline 270.51 & 79.5 & 6.29 & 6.44 & 1.01 & Fe XIV & 270.520 & 1.00 \\
271.98 & 12.0 & 6.15 & 6.25 & 0.83 & Si X & 271.992 & 0.99 \\
274.20 & 168.0 & 6.29 & 6.44 & 0.92 & Fe XIV & 274.203 & 0.99 \\
275.36 & 8.4 & 5.79 & 5.92 & 0.63 & Si VII & 275.361 & 1.00 \\
277.25 & 7.2 & 6.15 & 6.25 & 1.15 & Si X & 277.264 & 1.00 \\
278.26 & 2.4 & 6.32 & 6.46 & 0.71 & P XII & 278.286 & 0.99 \\
281.40 & 4.7 & 6.28 & 6.42 & 0.88 & S XI & 281.402 & 0.99 \\
284.15 & 2510.0 & 6.34 & 6.47 & 1.05 & Fe XV & 284.163 & 1.00 \\
285.82 & 6.9 & 6.27 & 6.41 & 0.94 & S XI & 285.822 & 0.98 \\
288.15 & 14.4 & 6.44 & 6.48 & 1.02 & Ni XVI & 288.165 & 0.98 \\
288.40 & 31.9 & 6.35 & 6.46 & 0.92 & S XII & 288.421 & 1.00 \\
\hline
\end{tabular}

"canonical" coronal and photospheric abundances, as shown in Table 2. We note that we obtained a sulfur abundance close to the photospheric value suggested by Asplund et al. (2009), which means that the $3 \mathrm{MK}$ loops had a FIP enhancement of 3.16. Sulphur has in fact a relatively high FIP and its abundance variations follow those of the higher-FIP elements. The abundances of $\mathrm{Ar}$ and $\mathrm{P}$ (two high-FIP elements) are also close to the values suggested by Asplund et al. (2009), although we note that photospheric measurements of argon do not exist, and recall that the P XII line is weak, so the P abundance is uncertain.

If we return now to the issue of the absolute iron abundance, we note that it could be higher than 8.0 dex (and the filling factor smaller than one), but then the abundances of the high-FIP elements would also have to be higher than photospheric. We also note that direct line-to-continuum measurements obtained from ground-based eclipse observations by Mason (1975) also suggest an increased calcium and iron abundance by a factor of about three, close to active regions. Mason (1975) measured the calcium abundance as 6.8 dex (i.e. only $15 \%$ lower than the value suggested here), and the iron abundance as 7.85 dex (only $30 \%$ lower than the present value).

\section{9. $\mathrm{Hi}-\mathrm{C}$}

The most direct way to address the issue of the existence (or not) of sub-resolution structures in active regions is to actually take images and spectra at higher resolution. Hi-C, during its successful flight (Cirtain et al. 2013), obtained coronal images of an active region at the highest resolution to date $\left(0.25^{\prime \prime}\right.$, about five times better than AIA) in a broad-band very similar to the AIA $193 \AA$ one. This band does not show the hot core loops, but is useful to discuss the 1.5-2.5 MK background emission, still unresolved at the AIA resolution. Hi-C could in principle also provide information on coronal loops that emit at these temperatures, but unfortunately the FOV only included the footpoints and legs of a few warm loops, and not their full extent. The AR observed by $\mathrm{Hi}-\mathrm{C}$ was very dispersed, had a large number of filaments, some very hot (10 MK) loops, and a large number of moss regions. The features discussed in Cirtain et al. (2013) were probably low-lying ones associated with a large filament, and not clearly connected with coronal loops.

A direct comparison of the Hi-C and AIA $193 \AA$ images immediately shows the much higher resolution of Hi-C, but only in the low-lying (e.g. not properly "coronal") moss regions, and in the low-temperature filament structures, since the prominencecorona transition region emits strongly in cool $(T<1 \mathrm{MK})$ lines observed by the $193 \AA$ band. Ground-based observations have already shown that filaments have a fine structure. The fine 
structure of the moss emission confirms the fine structure of the loops that are connected to it.

In all the other regions, there is very little difference between the Hi-C and AIA $193 \AA$ images, indicating that any unresolved emission in the AIA images is still unresolved at the Hi-C resolution. Some examples are given below.

First, we have processed the AIA full-Sun images, adjusting their plate scale and correcting for the stray light, as described previously. We then averaged the AIA images over a minute, at 18:55 UT. We took the simultaneous Hi-C level 1.5 data (as available form the Virtual Solar Observatory), and averaged the exposures obtained at 18:55:02, 18:55:08, and 18:55:13 UT. We applied the suggested rotation $(-1.81969$ degrees, keyword roll_angle), and then checked the co-alignment of the Hi-C data with the AIA $193 \AA$ image by plotting one image over the other one in a few sample moss areas. The moss areas are best for co-alignment because they are clear low-lying bright features. We found it necessary to apply a small shift of 1 " towards the $\mathrm{W}$ direction and $1.5^{\prime \prime}$ towards the $\mathrm{N}$ direction.

Considering the multi-thermal nature of the $193 \AA$ band discussed previously, a proper physical interpretation of the Hi-C $193 \AA$ images requires at least some DEM modelling. We therefore performed the DEM inversion on the full Hi-C FOV using the AIA data, adopting the present coronal abundances and a constant electron density of $2 \times 10^{9} \mathrm{~cm}^{-3}$, a typical average AR value.

We then selected three regions (R1, R2, and R3 shown in Fig. 12) where some coronal loop legs are present. Figure 13 shows region R1, which contains several loop legs that appear resolved in the $131 \AA$ (Fe VIII) and $171 \AA$ (Fe IX) images. The positions of seven cool loops are indicated with dashed lines in the profiles in Fig. 13. According to the DEM modelling, these cool loops are emitting in Fe VIII and Fe IX, and are still visible around $1 \mathrm{MK}$ (see the Fe $\mathrm{X}$ image), but at higher temperatures (see the Fe XII image) they disappear. Instead, other structures appear in the line of sight at temperatures at and above $1 \mathrm{MK}$. The strongest cool emission is concentrated between 10 and $25^{\prime \prime}$, where the DEM modelling predicts a depletion of the hotter Fe XII and especially Fe XIV. Such thermal structuring in active regions is common (although not ubiquitous). It was already seen in Skylab EUV observations (see, e.g. Foukal 1975) and SOHO CDS (Del Zanna \& Mason 2003), although with a much lower resolution $\left(5^{\prime \prime}\right)$.

The Fe XII and Fe XIV simulated images show a more diffuse, unresolved emission. The AIA and Hi-C $193 \AA$ images show both the resolved cool emission (mainly due to Fe VIII and Fe IX) and the unresolved 1-2 MK emission. It is interesting to note that there is very little difference in the AIA and Hi-C images, despite the factor of five higher resolution of $\mathrm{Hi}-\mathrm{C}$. This indicates that the hotter emission is not resolved even at the $\mathrm{Hi}-\mathrm{C}$ resolution, while the cooler emission is already resolved at the AIA resolution. This is not an instrumental effect, because the low-lying moss emission is clearly much better resolved by $\mathrm{Hi}-\mathrm{C}$ (cf. the lower right corner of the images in Fig. 13).

All the other coronal loops in the Hi-C FOV show similar features. As in the other ARs we have analysed, there are often locations where cooler and hotter loops are intermingled.

Figure 14 shows region R2, where six cool loops appear as almost resolved by AIA. Again, there is little difference between the Hi-C and AIA $193 \AA$ images. But in this case there is no clear anti-correlation between the locations of the cooler loops and the unresolved higher-temperature emission.

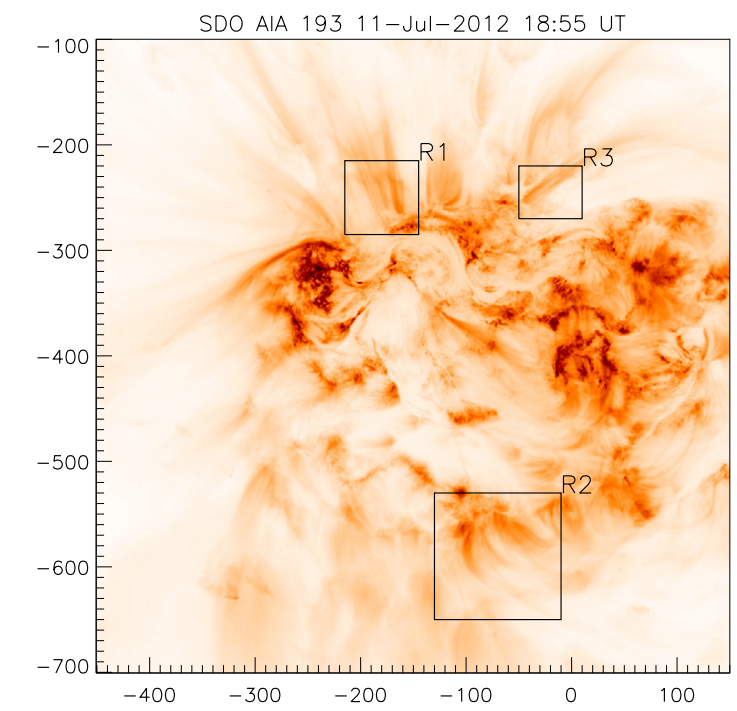

Fig. 12. Active region as observed in the AIA $193 \AA$ band during the $\mathrm{Hi}-\mathrm{C}$ rocket flight, with a few selected regions at the legs of several coronal loops.

Figure 15 shows region R3, on a very bright leg of a coronal loop. It is quite clear that the strong cool emission comprises at least three loops, visible in Fe VIII and Fe IX (AIA $171 \AA$ ). It is also quite clear that some fainter, different loops are present in the 1-2 MK range, as seen in Fe XII. These loops are not cospatial with the cooler ones and are visible in both the AIA and Hi-C $193 \AA$ images. In this case, these hotter loops appear as slightly better resolved in the Hi-C $193 \AA$ images.

\section{Summary and conclusions}

It is clear that significant advances in our understanding of the thermal structure in active regions can be achieved by combining high-resolution imaging with spectroscopy and the most recent atomic data. We have analysed several active regions, but shown here only a few selected examples. Clearly, not all active regions are the same, however several new important results have emerged from our small sample:

1) We have verified that our new Hinode EIS radiometric calibration is in very good agreement with the SDO AIA preflight and EVE in-flight calibrations. This gives us confidence in the present and future results. Some discrepancies are present, but they are within the combined uncertainties of the absolute calibrations of the AIA, EVE and EIS instruments (about $25 \%$ each).

2) We have verified that the atomic data we have provided for the EIS and AIA instruments are now accurate and sufficiently complete, with the exception of the AIA $211 \AA$ band.

3) We have confirmed the multi-thermal nature of the AIA EUV bands, also in quiescent AR observations. In the cores of ARs, only the 171 and $335 \AA$ bands are relatively isothermal. In the 193 and $211 \AA$ bands, only about half of the counts are due to Fe XII and Fe XIV. We have provided ways to use the AIA data to roughly estimate the various contributions. Significant continuum emission is present in the 94 and, especially, the $131 \AA$ band, confirming our previous suggestion (O'Dwyer et al. 2010). There is evidence that coronal abundances are more appropriate than the photospheric ones for the cores of active regions, but if the latter were the correct 

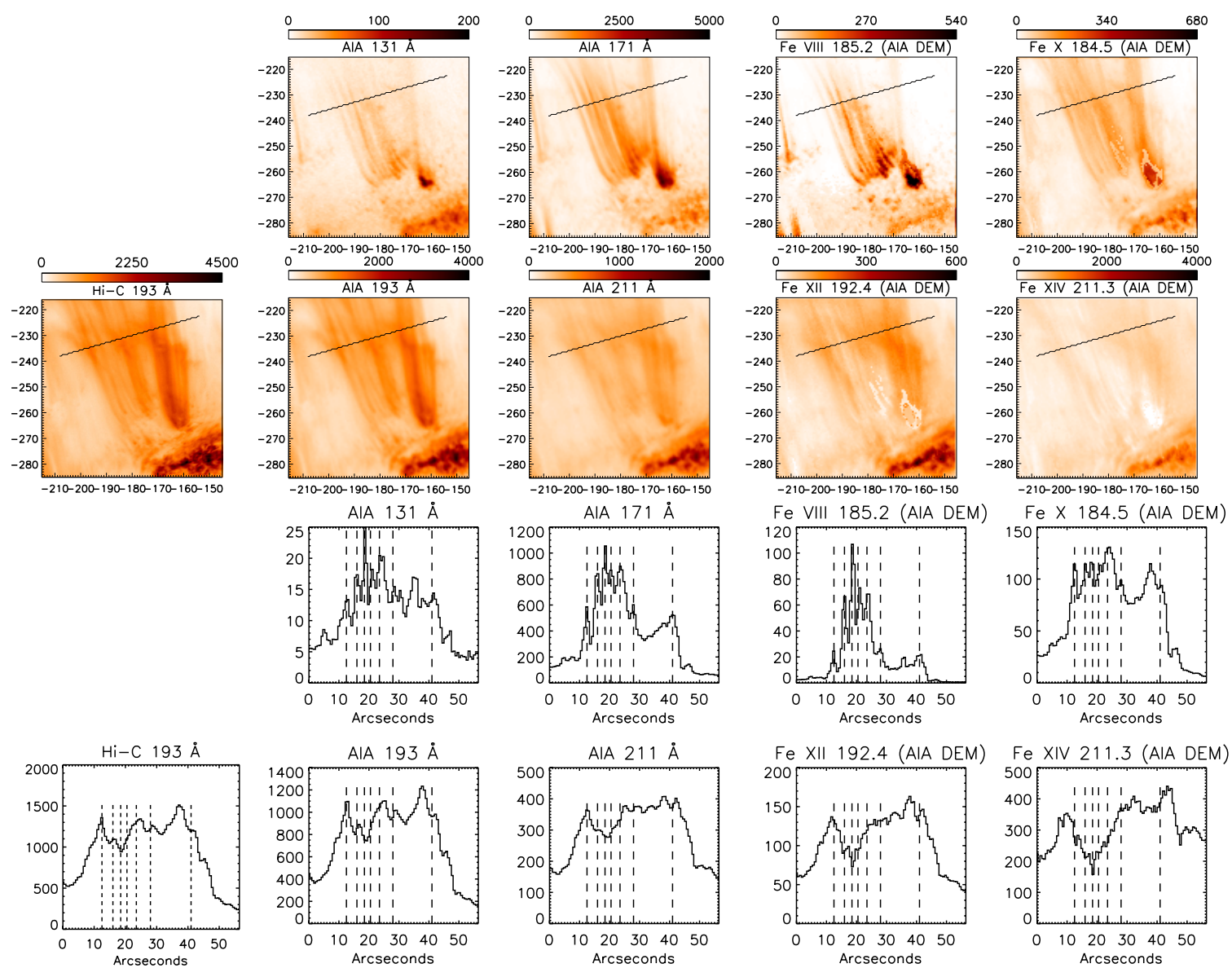

Fig. 13. Above: region R1, as observed by Hi-C, by AIA, and as predicted from the AIA DEM modelling. Below: profiles (averages over 1.8" in $\mathrm{DN} / \mathrm{s}$ ) along the segment indicated in the images. The positions of a few cool loops are noted with dashed lines.

ones, the continuum contribution to the $131 \AA$ band could be as high as $50 \%$. The discussion of the various contributions to the AIA bands, based on the EIS spectra and the atomic data, is key to the proper use of the AIA data for DEM modelling.

4) After two years of calculating new atomic data and in benchmarking these data, we have finally obtained a good agreement between observations and predictions for the AIA $94 \AA$ band. We confirm our (Del Zanna 2012a) suggestion that the newly identified Fe XIV 93.61 $\mathrm{A}$ line is the dominant contribution to the $94 \AA$ band in any AR observation, whenever Fe XVIII is not present. Studies of hot AR emission should take this into account.

5) We have shown the difficulty in estimating the Fe XVIII contribution directly with the DEM modelling (first method). A better way is to subtract the various contributions to the observed count rates in the $94 \AA$ band (second method). We used EIS spectroscopy and atomic data to provide a way to obtain rough estimates of the Fe XVIII emission in the $94 \AA$ band using only AIA data (third method). We showed that Fe XVIII emission is sometimes present in the cores of ARs, although this is often from $3 \mathrm{MK}$ and not $7 \mathrm{MK}$ plasma. The same applies to Fe XVII emission. These new results should be taken into account when using the $94 \AA$ band to diagnose the presence of very hot plasma. We showed the various complexities related to the calcium lines, and the surprising fact that Ca XVII is sometimes not observed when Fe XVIII is.
6) We have provided what are to our knowledge the first consistent measurements of electron densities and elemental abundances in a sample of 3 MK loops. They are significantly different than previous measurements. Low-FIP elements such as iron are enhanced by about a factor of 3, compared to the high-FIP elements. We performed several DEM analyses on a few ARs cores and obtained similar results. The path lengths obtained from the densities and emission measures indicate that the abundances of the low-FIP elements are enhanced, compared to the photospheric values, by at least a factor of 3, which implies spectroscopic filling factors close to 1 . If we assume that this is a real measure of how the plasma is distributed along the line of sight (see Judge 2000 for caveats), this suggests that we are still far from resolving the substructures in the 3 MK loops.

7) We have presented a new DEM method customised for analysing AIA observations. The comparison with direct spectroscopic observations from Hinode EIS is truly remarkable, providing confidence in using AIA for studying the thermal structure of ARs using the present method, atomic data, and caveats.

8) We often found, from the DEM modelling at the AIA resolution $\left(1^{\prime \prime}\right)$, that loops emitting at different temperatures are not co-spatial, as we also saw at much lower resolution with SOHO CDS (Del Zanna et al. 2006). To unambiguously identify the spatial location of all the loop structures visible along the line of sight would however probably require stereoscopic isothermal measurements. 

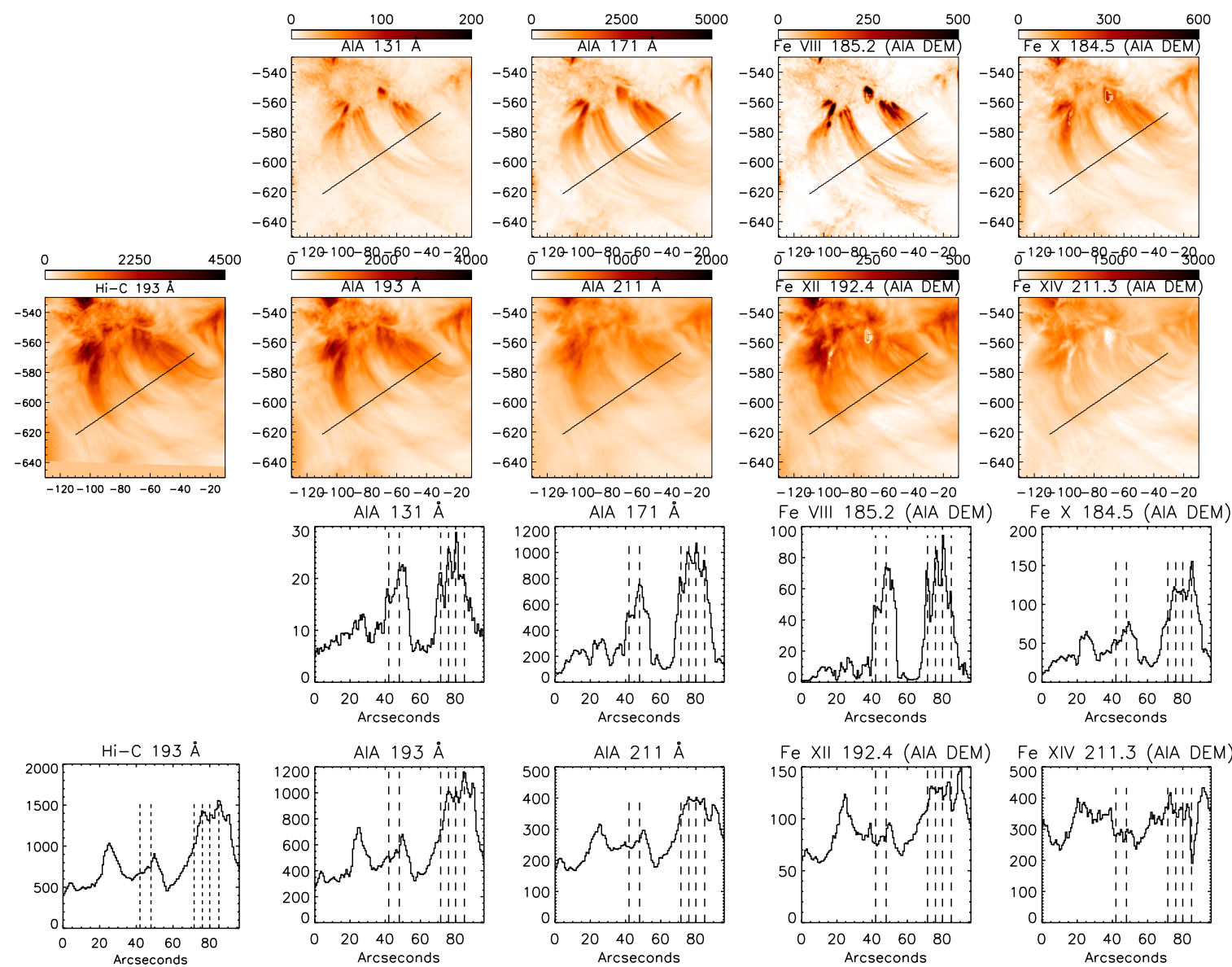

Fig. 14. Above: region R2, as observed Hi-C, by AIA, and as predicted from the AIA DEM modelling. Below: profiles (averages over 1.8" in $\mathrm{DN} / \mathrm{s}$ ) along the segment indicated in the images. The positions of a few cool loops are noted with dashed lines.

9) The AIA DEM modelling at $1^{\prime \prime}$ resolution indicates that warm loops (up to $1 \mathrm{MK}$ ) are nearly resolved, and have very little background/foreground emission. It also indicates that the emission at $1.5-2.5 \mathrm{MK}$ is progressively more unresolved. This phenomenon increases towards the core of ARs. This unresolved emission was found to comprise about $80 \%$ of the emission in TRACE and SOHO/CDS observations (Del Zanna \& Mason 2003). The DEM modelling from AIA suggests much lower values. We recall that this reduction is mainly due to the lower stray light of the AIA telescopes (compared to TRACE) and to their higher spatial resolution (compared to CDS and EIS).

The observations of the 2 MK unresolved emission in an active region (Del Zanna 2012b) showed increases by about a factor of 2-2.5 of the iron abundance, compared to say argon. The absolute values (i.e. relative to hydrogen) are not known, but if we assume, as in the case of the 3 MK loops, that it is the absolute iron abundance which increases, it follows that a factor of 2-3 enhancement in the observed emission is just due to this abundance variation and is not due to increased densities or temperatures.

In summary, the picture that is emerging is that of variable abundances within the different features in active regions. This is an important issue which complicates the interpretation of the observations, and that deserves further analysis.

10) We found very little difference in the AIA and Hi-C $193 \AA$ images of coronal structures, despite the factor of five higher resolution of Hi-C. The AIA DEM modelling indicates that there is cool emission (mainly due to Fe VIII and Fe IX) in the Hi-C images which appears to be already nearly resolved at the AIA resolution. The Hi-C images also contain hotter (1.5-2.5 MK) emission which in most of the places appears as unresolved as in the AIA images. This suggests that fundamental sub-structuring is well below the $0.25^{\prime \prime}$ resolution at these temperatures.

Understanding the multi-thermal structure of active regions is clearly a complex issue. One key issue that is currently debated in the literature regards the temporal evolution of the various structures. For example, are some of the warm loops hot $3 \mathrm{MK}$ loops observed while they are cooling? This is an even more complex issue that will be partly addressed in a separate publication, although it is really at the limits of current instrumentation.

Our SOHO CDS observations showed that the majority of the observed warm loops are long-lasting, i.e. do not appear to cool down or heat up significantly over timescales of hours (Del Zanna et al. 2006; Cirtain et al. 2007). Our results are in contrast to those obtained by Warren et al. (2003); Ugarte-Urra et al. (2006, 2009); Viall \& Klimchuk (2011), although they do not necessarily imply steady heating for the single structures. Indeed the higher SDO AIA resolution shows clear variations on timescales of minutes. The quasi-steady temperature structure of loops reflects the topology of the photospheric magnetic field which appears relatively steady over timescales of hours. This does not mean that the coronal magnetic field is steady. For example, according to our model of AR evolution (Del Zanna et al. 2011a), interchange reconnection should occur between the $3 \mathrm{MK}$ loops and the surrounding magnetic 

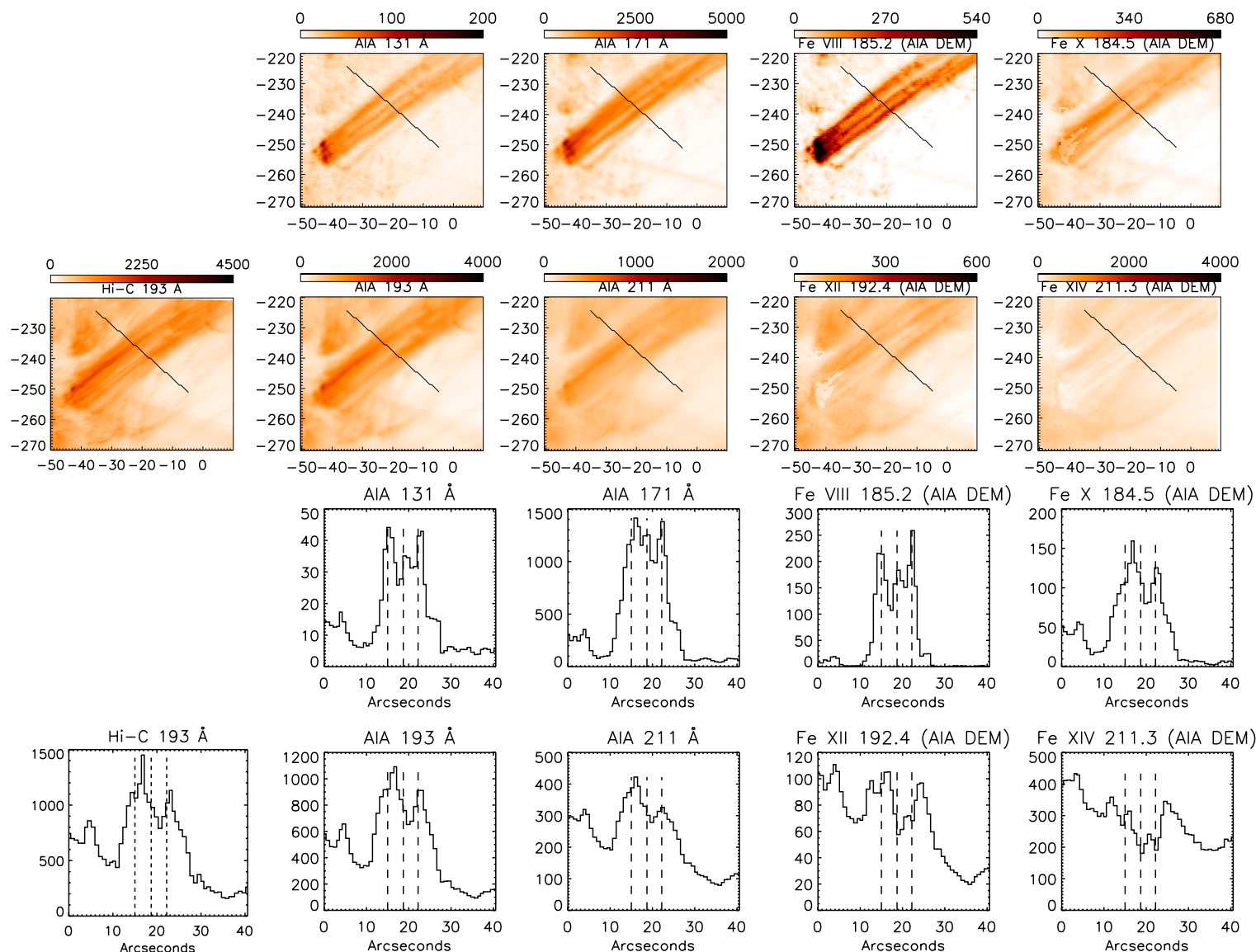

Fig. 15. Above: region R3, as observed Hi-C, by AIA, and as predicted from the AIA DEM modelling. Below: profiles (averages over 1.8" in $\mathrm{DN} / \mathrm{s}$ ) along the segment indicated in the images. The positions of a few cool loops are noted with dashed lines.

field, producing as a byproduct the formation of warm loops. This probably occurs on very short timescales and small spatial scales, well below the resolution of current instrumentation. It is however a long-lasting (hours to days) quasi-steady process.

The other key issue that is currently debated regards the subresolution structures. We believe that significant advances in the future can only be obtained with high-resolution spectroscopy (e.g. the proposed Solar-C spectrometer, see Teriaca et al. 2012) and isothermal imaging. The current imaging at $1^{\prime \prime}$ (AIA) and $0.25^{\prime \prime}$ (Hi-C) resolution indicates, however, that loops at temperatures above $1.5 \mathrm{MK}$ are still far from being resolved. We suggest that future high-resolution imaging with $\mathrm{Hi}-\mathrm{C}$ is done with isothermal bands, possibly covering the $0.7-1.5 \mathrm{MK}$ temperature range.

Acknowledgements. Support from STFC (UK) is acknowledged. The referee is thanked for constructive and detailed comments which helped to improve this rather long paper. H.E. Mason is thanked for useful discussions and comments. I. Ugarte-Urra and H. Warren are thanked for clarifications on their procedure to estimate Fe XVIII from the AIA $94 \AA$ data. CHIANTI is a collaborative project involving researchers at the Universities of Cambridge (UK), George Mason, Michigan (USA). The work of the UK APAP Network was funded by the UK STFC under grant No. PP/E001254/1 with the University of Strathclyde. Hinode is a Japanese mission developed and launched by ISAS/JAXA, with NAOJ as domestic partner and NASA and STFC (UK) as international partners. It is operated by these agencies in co-operation with ESA and NSC (Norway). We acknowledge the High resolution Coronal Imager instrument team for making the flight data publicly available. MSFC/NASA led the mission and partners include the Smithsonian Astrophysical Observatory in Cambridge, Mass.; Lockheed Martin's Solar Astrophysical Laboratory in Palo Alto, Calif.; the University of Central Lancashire in Lancashire, England; and the Lebedev Physical Institute of the Russian Academy of Sciences in Moscow.

\section{References}

Aschwanden, M. J., \& Boerner, P. 2011, ApJ, 732, 81

Asplund, M., Grevesse, N., Sauval, A. J., \& Scott, P. 2009, ARA\&A, 47, 481

Boerner, P., Edwards, C., Lemen, J., et al. 2011, Sol. Phys., 193

Brickhouse, N. S., \& Schmelz, J. T. 2006, ApJ, 636, L53

Cirtain, J. W., Del Zanna, G., DeLuca, E. E., et al. 2007, ApJ, 655, 598

Cirtain, J. W., Golub, L., Winebarger, A. R., et al. 2013, Nature, 493, 501

Craig, I. J. D., \& Brown, J. C. 1976, A\&A, 49, 239

Craig, I. J. D., \& Brown, J. C. 1986, in Inverse problems in astronomy: A guide to inversion strategies for remotely sensed data, Adam Hilger, Ltd., 159

Del Zanna, G. 1999, Ph.D. Thesis, Univ. of Central Lancashire, UK

Del Zanna, G. 2003a, A\&A, 406, L5

Del Zanna, G. 2003b, A\&A, 406, L5

Del Zanna, G. 2006, A\&A, 459, 307

Del Zanna, G. 2008, A\&A, 481, L69

Del Zanna, G. 2009a, A\&A, 508, 501

Del Zanna, G. 2009b, A\&A, 508, 513

Del Zanna, G. 2012a, A\&A, 546, A97

Del Zanna, G. 2012b, A\&A, 537, A38

Del Zanna, G. 2013, A\&A, 555, A47

Del Zanna, G., \& Ishikawa, Y. 2009, A\&A, 508, 1517

Del Zanna, G., \& Mason, H. E. 2003, A\&A, 406, 1089

Del Zanna, G., \& Storey, P. J. 2013, A\&A, 549, A42

Del Zanna, G., \& Woods, T. N. 2013, A\&A, 555, A59

Del Zanna, G., Bromage, B. J. I., \& Mason, H. E. 2001, in Solar and Galactic Composition, AIP Conf. Proc., 598, 59

Del Zanna, G., Landini, M., \& Mason, H. E. 2002, A\&A, 385, 968

Del Zanna, G., Bromage, B. J. I., \& Mason, H. E. 2003, A\&A, 398, 743

Del Zanna, G., Berrington, K. A., \& Mason, H. E. 2004, A\&A, 422, 731

Del Zanna, G., Mason, H. E., \& Cirtain, J. 2006, in SOHO-17. 10 Years of SOHO and Beyond, ESA SP, 617, 86

Del Zanna, G., Andretta, V., Chamberlin, P. C., Woods, T. N., \& Thompson, W. T. 2010a, A\&A, 518, A49

Del Zanna, G., Storey, P. J., \& Mason, H. E. 2010b, A\&A, 514, A40 
Del Zanna, G., Aulanier, G., Klein, K.-L., \& Török, T. 2011a, A\&A, 526, A137 Del Zanna, G., Mitra-Kraev, U., Bradshaw, S. J., Mason, H. E., \& Asai, A. 2011b, A\&A, 526, A1

Del Zanna, G., O’Dwyer, B., \& Mason, H. E. 2011c, A\&A, 535, A46

Del Zanna, G., Storey, P. J., Badnell, N. R., \& Mason, H. E. 2012a, A\&A, 543, A139

Del Zanna, G., Storey, P. J., Badnell, N. R., \& Mason, H. E. 2012b, A\&A, 541, A90

Dere, K. P., Landi, E., Young, P. R., et al. 2009, A\&A, 498, 915

Edlén, B. 1937, Z. Astrophys., 104, 407

Feldman, U., Mandelbaum, P., Seely, J. F., Doschek, G. A., \& Gursky, H. 1992, ApJS, 81, 387

Foster, A. R., \& Testa, P. 2011, ApJ, 740, L52

Foukal, P. 1975, Sol. Phys., 43, 327

Griffin, D. C., Pindzola, M. S., \& Badnell, N. R. 2000, A\&AS, 142, 317

Hannah, I. G., \& Kontar, E. P. 2012, A\&A, 539, A146

Judge, P. G. 2000, ApJ, 531, 585

Judge, P. G. 2010, ApJ, 708, 1238

Judge, P. G., Hubeny, V., \& Brown, J. C. 1997, ApJ, 475, 275

Kashyap, V., \& Drake, J. J. 1998, ApJ, 503, 450

Klimchuk, J. A. 2006, Sol. Phys., 234, 41

Klimchuk, J. 2010, in COSPAR Meeting 38, 38th COSPAR Scientific Assembly, 2897

Landi, E., \& Dere, K. P. 2013, ADNDT, submitted

Landi, E., \& Klimchuk, J. A. 2010, ApJ, 723, 320

Landi, E., Young, P. R., Dere, K. P., Del Zanna, G., \& Mason, H. E. 2013, ApJ, 763,86

Lemen, J. R., Title, A. M., Akin, D. J., et al. 2012, Sol. Phys., 275, 17

Liang, G. Y., Whiteford, A. D., \& Badnell, N. R. 2009, A\&A, 500, 1263

Liang, G. Y., Badnell, N. R., Crespo López-Urrutia, J. R., et al. 2010, ApJS, 190, 322

Lodders, K., Palme, H., \& Gail, H. 2009, in Abundances of the elements in the Solar System, ed. Landolt Börnstein, 712

Manson, J. E. 1972, Sol. Phys., 27, 107

Martens, P. C. H., Kankelborg, C. C., \& Berger, T. E. 2000, ApJ, 537, 471

Mason, H. E. 1975, MNRAS, 171, 119

O’Dwyer, B., Del Zanna, G., Mason, H. E., Weber, M. A., \& Tripathi, D. 2010, A\&A, 521, A21

O’Dwyer, B., Del Zanna, G., Badnell, N. R., Mason, H. E., \& Storey, P. J. 2012, A\&A, 537, A22

Peres, G., Reale, F., \& Golub, L. 1994, ApJ, 422, 412

Petkaki, P., Del Zanna, G., Mason, H. E., \& Bradshaw, S. 2012, A\&A, 547, A25
Poduval, B., DeForest, C. E., Schmelz, J. T., \& Pathak, S. 2013, ApJ, 765, 144 Reale, F. 2010, Living Rev. Sol. Phys., 7, 5

Reale, F., Guarrasi, M., Testa, P., et al. 2011, ApJ, 736, L16

Rosner, R., Tucker, W. H., \& Vaiana, G. S. 1978, ApJ, 220, 643

Schmelz, J. T., Saba, J. L. R., Ghosh, D., \& Strong, K. T. 1996, ApJ, 473, 519

Schmelz, J. T., Nasraoui, K., Rightmire, L. A., et al. 2009, ApJ, 691, 503

Schmelz, J. T., Kimble, J. A., Jenkins, B. S., et al. 2010, ApJ, 725, L34

Schmelz, J. T., Jenkins, B. S., Worley, B. T., et al. 2011, ApJ, 731, 49

Schmelz, J. T., Reames, D. V., von Steiger, R., \& Basu, S. 2012, ApJ, 755, 33

Schmelz, J. T., Pathak, S., Jenkins, B. S., \& Worley, B. T. 2013, ApJ, 764, 53

Schrijver, C. J., Title, A. M., Berger, T. E., et al. 1999, Sol. Phys., 187, 261

Storey, P. J., Zeippen, C. J., \& Le Dourneuf, M. 2002, A\&A, 394, 753

Strong, K. 1978, Ph.D. Thesis, University College London, UK

Tayal, S. S., \& Zatsarinny, O. 2011, ApJ, 743, 206

Teriaca, L., Warren, H. P., \& Curdt, W. 2012, ApJ, 754, L40

Testa, P., \& Reale, F. 2012, ApJ, 750, L10

Testa, P., De Pontieu, B., Martínez-Sykora, J., Hansteen, V., \& Carlsson, M. 2012a, ApJ, 758, 54

Testa, P., Drake, J. J., \& Landi, E. 2012b, ApJ, 745, 111

Tripathi, D., Mason, H. E., Dwivedi, B. N., del Zanna, G., \& Young, P. R. 2009 ApJ, 694, 1256

Tripathi, D., Mason, H. E., Del Zanna, G., \& Young, P. R. 2010, A\&A, 518, A42

Tripathi, D., Klimchuk, J. A., \& Mason, H. E. 2011, ApJ, 740, 111

Tripathi, D., Mason, H. E., \& Klimchuk, J. A. 2012, ApJ, 753, 37

Ugarte-Urra, I., Winebarger, A. R., \& Warren, H. P. 2006, ApJ, 643, 1245

Ugarte-Urra, I., Warren, H. P., \& Brooks, D. H. 2009, ApJ, 695, 642

Veck, N. J., Strong, K. T., Jordan, C., et al. 1984, MNRAS, 210, 443

Viall, N. M., \& Klimchuk, J. A. 2011, ApJ, 738, 24

Wang, T., Thomas, R. J., Brosius, J. W., et al. 2011, ApJS, 197, 32

Warren, H. P., Winebarger, A. R., \& Mariska, J. T. 2003, ApJ, 593, 1174

Warren, H. P., Ugarte-Urra, I., Doschek, G. A., Brooks, D. H., \& Williams, D. R. 2008, ApJ, 686, L131

Warren, H. P., Winebarger, A. R., \& Brooks, D. H. 2012, ApJ, 759, 141

Widing, K. G., \& Feldman, U. 1989, ApJ, 344, 1046

Widing, K. G., \& Sandlin, G. D. 1968, ApJ, 152, 545

Winebarger, A. R., Schmelz, J. T., Warren, H. P., Saar, S. H., \& Kashyap, V. L. 2011, ApJ, 740, 2

Witthoeft, M. C., \& Badnell, N. R. 2008, A\&A, 481, 543

Witthoeft, M. C., Badnell, N. R., Del Zanna, G., Berrington, K. A., \& Pelan, J. C. 2006, A\&A, 446, 361

Woods, T. N., Eparvier, F. G., Hock, R., et al. 2012, Sol. Phys., 275, 115

Young, P. R., Del Zanna, G., Mason, H. E., et al. 2007, PASJ, 59, 857 